International Journal of Neural Systems, Vol. 29, No. 6 (2019) 1850055 (26 pages)

(C) The Author(s)

DOI: 10.1142/S0129065718500557

\title{
Cortical Thickness and Surface Area Networks in Healthy Aging, Alzheimer's Disease and Behavioral Variant Fronto-Temporal Dementia
}

\author{
Vesna Vuksanović* \\ Aberdeen Biomedical Imaging Center \\ University of Aberdeen \\ Aberdeen, AB25 2ZD, UK \\ vesna.vuksanovic@abdn.ac.uk \\ Roger T Staff and Trevor Ahearn \\ Imaging Physics, National Health Service Grampian \\ Aberdeen, AB25 2ZD, UK \\ Alison D Murray \\ Aberdeen Biomedical Imaging Center \\ University of Aberdeen \\ Aberdeen, AB25 2ZD, UK \\ Claude M Wischik \\ TauRx, Therapeutics, Aberdeen, AB24 5RP, UK \\ School of Medicine and Dentistry, University of Aberdeen \\ Aberdeen, AB25 2ZD, UK
}

Accepted 5 November 2018

Published Online 8 January 2019

\begin{abstract}
Models of the human brain as a complex network of inter-connected sub-units are important in helping to understand the structural basis of the clinical features of neurodegenerative disorders. The aim of this study was to characterize in a systematic manner the differences in the structural correlation networks in cortical thickness (CT) and surface area (SA) in Alzheimer's disease (AD) and behavioral variant Fronto-Temporal Dementia (bvFTD). We have used the baseline magnetic resonance imaging (MRI) data available from a large population of patients from three clinical trials in mild to moderate AD and mild bvFTD and compared this to a well-characterized healthy aging cohort. The study population comprised 202 healthy elderly subjects, 213 with bvFTD and 213 with AD. We report that both CT and SA network architecture can be described in terms of highly correlated networks whose positive and inverse links map onto the intrinsic modular organization of the four cortical lobes. The topology of the disturbance in structural network is different in the two disease conditions, and both are different from normal aging. The changes from normal are global in character and are not restricted to fronto-temporal and temporoparietal lobes, respectively, in bvFTD and AD, and indicate an increase in both global correlational strength and in particular nonhomologous inter-lobar connectivity defined by inverse correlations. These inverse correlations appear to be adaptive in character, reflecting coordinated increases in CT and SA that may compensate for corresponding impairment in functionally linked nodes. The effects were more pronounced in the cortical thickness atrophy network in bvFTD and in the surface area network in AD. Although lobar modularity is preserved in the context of neurodegenerative disease, the hub-like
\end{abstract}

${ }^{*}$ Corresponding author.

This is an Open Access article published by World Scientific Publishing Company. It is distributed under the terms of the Creative Commons Attribution 4.0 (CC-BY) License. Further distribution of this work is permitted, provided the original work is properly cited. 
organization of networks differs both from normal and between the two forms of dementia. This implies that hubs may be secondary features of the connectivity adaptation to neurodegeneration and may not be an intrinsic property of the brain. However, analysis of the topological differences in hub-like organization $\mathrm{CT}$ and SA networks, and their underlying positive and negative correlations, may provide a basis for assisting in the differential diagnosis of bvFTD and AD.

Keywords: Structural correlation networks; behavioral variant frontotemporal dementia; Alzheimer's disease.

\section{Introduction}

Models of the human brain as a complex network of interconnected sub-units have improved understanding of normal brain organization ${ }^{11}$ and have made it possible to address functional changes in neurological disorders. ${ }^{[2}$ These sub-units constitute so-called brain modules, i.e. sub-sets of regions that have a high density of connections within the same sub-set and a lower density of connections between sub-sets. It is thought that this modular organization underpins efficient integration of information between spatially segregated neural processes necessary to support diverse cognitive and behavioral functions. $\frac{3}{3}$ Cognitive and behavioral symptoms in patients with dementia are considered to arise from a disruption in the neuronal interactions required for normal cognitive integration. ${ }^{\sqrt{6}}$ Changes in functional brain networks inferred from fMRI or PET data have been found to distinguish behavioral variant frontotemporal dementia (bvFTD) 7 from Alzheimer's disease $(\mathrm{AD})$ and mild cognitive impairment $(\mathrm{MCI})^{\sqrt[819]{1}}$ in small studies in research settings. Similarly, longitudinal changes in MEG and EEG networks have been explored over short periods. ${ }^{10111}$ The opposite is also true, in that the changes seen in disease may shed light on important properties of large-scale organization in the normal brain. ${ }^{12}$ Since fMRI and PET are not routinely available in clinical practice, we have sought to determine whether a similar structural network approach applied to routine MRI data could be used to distinguish different clinical forms of dementia.

Previous studies of structural networks in health and disease have examined pair-wise regional correlations in cortical volume and thickness using T1weighted magnetic resonance images (MRI) $\frac{13}{13}$ This approach has shown potential clinical relevance using regional volume data from patients with schizophrenia. ${ }^{14}$ However, volumetric measures, which represent the product of cortical thickness (CT) and surface area (SA), may confound more subtle underlying differences. For example, consideration of changes in cortical thickness may provide insight into how disease alters the size, density and arrangement of the cells within cortical layers 15 Changes in surface area, on the other hand, may provide information regarding disturbance in integration between groups of columns in disease ${ }^{16}$ Earlier studies have suggested that separate consideration of thickness and area may be more informative in the context of Parkinson's disease ${ }^{17}$ and in identifying subtle differences between bvFTD and AD! ${ }^{18}$ So far, the correlational network organization of regional thickness and surface area have been investigated only in healthy subjects 19 Recent neuroimaging studies have also shown that large-scale anatomical connectivity in the human brain is inherently modular in its organization ${ }^{20[21}$ and that cortical thickness networks change during brain maturation and development $\stackrel{22}{\text { It }}$ is also known that cortical atrophy mirrors the lobespecific spatial organization of the areas involved in the later stages of $\mathrm{AD}$ and bvFTD, with characteristic fronto-temporal atrophy in bvFTD and temporoparietal atrophy in $\mathrm{AD}$ reported consistently in numerous studies.23 It would be expected that topological measures of structural network organization would also be affected in a lobe-specific manner.

In the present study, we have examined the topological organization of cortical thickness and surface area networks in a clinical population of patients with bvFTD and AD available from three global Phase 3 clinical trials. Two of these were in mild to moderate $\mathrm{AD}^{2425}$ and the third was from the largest study in bvFTD conducted to date ${ }^{26}$ Because MRI data were collected at 3-monthly intervals in all three trials according to standardized protocols and quality control measures, it was possible to undertake systematic comparisons of network organization in the two forms of dementia using MRI scans collected at baseline. Comparable data were available 
from well characterized healthy elderly subjects participating in an ongoing longitudinal study of the Aberdeen 1936 Birth Cohort. ${ }^{27}$ In all, data were available in 628 subjects with 213 in each of the dementia groups and 202 normal elderly subjects. This study therefore represents the largest comparative study to date of the structural network organization of the cortex in these three contexts. The approach we have taken is to analyze positive and inverse correlations (or network edges) in cortical thickness and surface area in 68 cortical brain regions to define the topological organization of structural networks in terms of lobar modularity. We report that the disturbances in structural network organization are not restricted to fronto-temporal and temporo-parietal lobes, respectively, in bvFTD and $\mathrm{AD}$ but are global in nature and that their hub-like topology is altered in both conditions. An important change distinguishing both forms of dementia from normal elderly controls is the emergence of significant inverse correlational networks linking anterior and posterior brain regions which may relate to functional adaptations or compensations for impairment due to pathology.

\section{Methods}

\subsection{Participants}

A total of 628 participants (202 healthy elderly (HE), 213 bvFTD and 213 AD) were included in this study. bvFTD and AD patients were recruited as a part of multinational, randomized, controlled, clinical trials (clinicaltrials.gov; TRx-237-005, TRx237-007 and TRx-237-015). Only the baseline visit data, obtained prior to pharmaceutical intervention, were used in the current study. In short, bvFTD patients were diagnosed according to the International Consensus Criteria for bvFTD, 28 with mild severity on the Mini-Mental State Examination (MMSE) score of 20-30 inclusive. To match the number of subjects available from the limiting bvFTD group, $213 \mathrm{AD}$ subjects were randomly drawn from a much larger $\mathrm{AD}$ pool $(N=1132)$ available from studies TR-237-005 and TRx-237-015. AD patients were diagnosed according to criteria from the National Institute of Aging and the Alzheimer's Association, with mild to moderate severity defined by an MMSE score of 14-26 (inclusive) and a Clinical Dementia Rating (CDR) total score of 1 or 224/25 HE subjects $(N=202)$ were also selected from a larger available pool of 244 subjects from a well characterized Aberdeen 1936 Birth Cohort (ABC36)!27 Details and the supporting information for demographic, clinical or neuroimaging characteristics are provided in Table 1 .

\subsection{Data acquisition and processing}

Multi-site data from trial patients were pooled to permit overall group-wise statistical comparisons. The data used were standard T1-weighted MR images acquired using equivalent manufacturer sequences. The trial scanners were limited to $1.5 \mathrm{~T}$ and $3 \mathrm{~T}(30 \%)$ field strengths from the three major manufactures (Philips, GE and Siemens). MRI images in the ABC36 cohort were all acquired using the same (Philips) 3T scanner. The images were processed using a standard automated processing pipeline implemented in FreeSurfer v5.3.0, which is freely available for download from http://surfer.nmr.mgh.harvard.edu/. In addition to

Table 1. Demographic, cognitive and cortico-morphological characteristics of participants.

\begin{tabular}{lllllll}
\hline & $\begin{array}{c}\text { M/F } \\
\text { mean(sd) }\end{array}$ & $\begin{array}{c}\text { Age (yrs) } \\
\text { mean(sd) }\end{array}$ & $\begin{array}{c}\text { EDU (yrs) } \\
\text { mean(sd) }\end{array}$ & $\begin{array}{c}\text { MMSE } \\
\text { mean(sd) }\end{array}$ & $\begin{array}{c}\text { CT (mm) } \\
\text { mean(sd) }\end{array}$ & $\begin{array}{c}\text { Total SA }\left(\times 10^{5} \mathrm{~mm}^{2}\right) \\
\text { mean (sd) }\end{array}$ \\
\hline HE & $106 / 95$ & $64(1)^{\mathrm{b}}$ & $11(1)$ & $29(1)^{\mathrm{a}, \mathrm{b}}$ & $2.43(0.1)^{\mathrm{a}, \mathrm{b}}$ & $106.9(1.4)^{\mathrm{a}, \mathrm{b}}$ \\
bvFTD & $136 / 77^{\mathrm{a}, \mathrm{c} *}$ & $64(8)^{\mathrm{c}}$ & $12(7)$ & $24(4)^{\mathrm{c}}$ & $2.20(0.15)$ & $102.8(1.5)$ \\
AD & $105 / 108$ & $71(10)$ & $16(13)$ & $21(4)$ & $2.18(0.15)$ & $102.5(1.6)$ \\
\hline
\end{tabular}

Notes: Abbreviations: HE - healthy elderly, bvFTD - behavioral variant frontotemporal dementia, AD - Alzheimers disease, M - males, F - females, EDU - age at leaving full time education, MMSE - Mini-Mental-State Examination, CT - cortical thickness, SA - surface area. Significant differences between groups: $\mathrm{a}-\mathrm{HE} / \mathrm{bvFTD}, \mathrm{b}-\mathrm{HE} / \mathrm{AD}, \mathrm{c}-\mathrm{bvFTD} / \mathrm{AD}(p<0.05)$; $^{*}$ - results reported for the chi-square test. 
widely used volume-based methods of image processing, the FreeSurfer pipeline produces surface-based regional measurements of cortical morphology such as thickness, the local curvature or surface area. The technical details of these procedures are described elsewhere $29 \sqrt{31}$ Surface-based methods of estimation of cortical morphology are able to account for the complex shape and folding of the cortical surface providing better inter-individual alignment which aids comparisons across individuals. 30

$\mathrm{CT}$ and SA measurements, considered in this study, were calculated according to the following procedures. SA was calculated using triangular tessellation of the gray/white matter interface and white matter/cerebrospinal fluid boundary (pial surface) ${ }^{29}$ $\mathrm{CT}$ was calculated as an average of the distance from the white matter surface to the closest point on the pial surface and from that point back to the closest point to the white matter surface. ${ }^{30}$ We used the FreeSurfer parcellation scheme (aparc.lh/rh), based on the Desikan-Killiany Atlas, ${ }^{32}$ to extract $\mathrm{CT}$ and SA of 68 cortical regions from both hemispheres. A list of regions and their lobar assignment is given in Table B.1 Previous multi-site MRI studies have reported reliability of the cortical surfacebased regional measurements given the same image processing technique. ${ }^{33}$ Slightly biased results for the CT measurements across different magnetic field strengths have also been reported ${ }^{33 / 34}$ All data presented here were raw, uncorrected cortical thickness/surface area data from the FreeSurfer v5.3.0 pipeline. However, correcting for scanner strength as an additional controlling variable in CT measurements had no effect on the resulting correlation structure of the networks.

\subsection{Structural correlation network construction}

A structural correlation network, in which a cortical region represents a node and a pair-wise correlation between nodes represents a graph edge or link/connection, was constructed correlating either $\mathrm{SA}$ or $\mathrm{CT}$ across all participants within each diagnostic category. Each correlation matrix was calculated based on $S \times N$ array containing $N$ regional $\mathrm{CT} / \mathrm{SA}$ values on $S$ subjects within each group. In this way six $N \times N$ (i.e. $68 \times 68$ ) correlation matrices were obtained (one CT or SA structural correlation matrix for each study group; see also Fig. (1).
The matrix element $e_{i j}$ is the value of the partial correlation between the regions $i$ and $j(i, j=$ $1,2, \ldots, N)$ (i.e. between vectors $\mathbf{x}_{i}$ and $\mathbf{x}_{j}$ that contain regional measurements from subjects within each group). The partial correlations were calculated as linear, Pearson's correlation coefficient between pairs of $\mathbf{x}_{i}$ and $\mathbf{x}_{j}$, after first removing the effects of all other regions $m \neq(i, j)$ and then adjusting both $\mathbf{x}_{i}$ and $\mathbf{x}_{j}$ for controlling variables (stored in a separate array $S \times C$, where $C$ represents number of controlling variables) $\stackrel{35}{5}$ This means that prior to the correlation analysis a linear regression was performed on every $\mathbf{x}_{i}$ to remove the effect of age, gender and mean CT (mean cortical thickness of all areas) or total SA (sum of overall surface areas)!19] Selfcorrelations (represented by the main matrix diagonal) were excluded from the analysis and the network measures were calculated on the lower triangular part of the matrix. The partial correlation, $e_{i j}$ (i.e. edge weight), can be calculated according to the following general equations:

$$
e_{i j}=\rho_{i j \cdot c} \equiv \operatorname{corr}\left(x_{i}, x_{j} \mid x_{c}\right),
$$

where $x_{i / j}$ denotes an array of variables and $x_{c}$ denotes any subset of conditioning variables. To arrive at this general form of the partial correlation we start from $i, j, c=1,2,3$

$$
\rho_{12 \cdot 3}=\frac{\rho_{12}-\rho_{13} \rho_{23}}{\left(1-\rho_{13}^{2}\right)\left(1-\rho_{23}^{2}\right)^{1 / 2}} .
$$

Hence, for any subset $c$ of conditioning variables we will have

$$
\rho_{12 \cdot 3 c}=\frac{\rho_{12 \cdot c}-\rho_{13 \cdot c} \rho_{23 \cdot c}}{\left(1-\rho_{13 \cdot c}^{2}\right)\left(1-\rho_{23 \cdot c}^{2}\right)^{1 / 2}} .
$$

To verify that each network retained only statistically significant correlations, the calculated correlation coefficients were adjusted for multiple tests using the False Discovery Rate (FDR) procedure. ${ }^{36}$ The FDR procedure tests each $p$-value against a corrected significance level $(\alpha=0.05)$ and accepts only $p$-values smaller than the adjusted significance level as truly significant. Those pair-wise correlations that did not pass the FDR test were set to zero; otherwise, all nonzero correlations, whether positive or negative, were retained (see Fig. A.1). In this way, a $68 \times 68$ correlation matrix was constructed representing the 'structural correlation network' for either SA or CT. A matrix element quantifies the strength of the correlation between cortical regions for $\mathrm{CT}$ or 
SA and it does not in itself represent an actual physical connection. In the context of structural correlation network analysis in neurodegenerative disorders, such correlations imply either a co-atrophy relationship (if positive) or an inverse atrophy/hypertrophy relationship (if negative) between brain regions.

\subsection{Network analysis}

We used the following network measures to compare the structural network properties of the three clinical groups: edge strength, node degree, node withinmodule degree $z$-score and participation index. Edge strength and node degree represent two basic network attributes; they respectively quantify the correlation strength between nodes and the number of pairwise correlations for each node. To assess whether cortical lobes represent modules we utilized two network measures which assess modularity in network interactions, namely within-module degree $z$-score and participation index. All measures (except node degree) were computed on weighted graphs and were estimated as averages across the four lobes (as described below). From theoretical studies it is known that the calculated topological properties of a network depends on the choice of the threshold value $\frac{37}{}$ For the present study, we chose a fixed threshold for each group-based correlation matrix, similar to the approach taken by Vaśa and colleges 22 Our main focus in this study was to assess how different clinically defined neurodegenerative disorders impact on structural network organization as defined in terms of $\mathrm{CT}$ and $\mathrm{SA}$ correlations.

Node degree: Node degree $\left(k_{i}\right)$ represents the number of significant correlations for each node in the network. In general, node degree is calculated from a binarized correlation matrix where each significant correlation in the matrix is replaced with either 1 if it is significant or with 0 if it is not. The degree of a node $i$ (i.e. the number of significant links connected to the node) was calculated as

$$
k_{i}=\sum_{j=1}^{N} a_{i j},
$$

where $N$ is the total number of nodes and $a_{i j}$ represents connection between nodes $i$ and $j: a_{i j}=1$, if there is a direct connection between nodes and $a_{i j}=0$ otherwise.
Modularity index: Node participation index and within-module degree $z$-score assesses the role of a node according to modules. Network modules (also called community structures) represent densely connected subgraphs of a network, i.e. subsets of nodes within which network connections are denser, and between which connections are sparser. Our aim was to examine the modular organization of frontal, temporal, parietal and occipital divisions of $\mathrm{CT}$ or SA networks defined as modules. Since these lobar divisions of the cortical surface need not be modular in themselves, it was first necessary to test whether lobar divisions are intrinsically modular. This can be done by calculating modularity index $(Q)$ of the networks according to lobe. The modularity index quantifies the observed fraction of within- module degree values relative to those expected if connections were randomly distributed across the network. Since the constructed CT/SA network contains both positive and negative edge strengths, we used the asymmetric generalization of the modularity quality function introduced in Rubinov and Sporns 38 .

$$
\begin{aligned}
Q= & \frac{1}{\nu^{+}} \sum_{i j}^{N}\left(\omega_{i j}{ }^{+}-e_{i j}{ }^{+}\right) \delta_{M_{i}, M_{j}} \\
& -\frac{1}{\nu^{+}+\nu^{-}} \sum_{i j}^{N}\left(\omega_{i j}{ }^{-}-e_{i j}{ }^{-}\right) \delta_{M_{i}, M_{j}},
\end{aligned}
$$

where $\omega_{i j}{ }^{+}$is equal to the $i, j$ th element of the correlation matrix (i.e. strength of the pair-wise correlation between cortical regions) $\omega_{i j}$ if $\omega_{i j}>0$ and is equal to zero, otherwise. Similarly, $\omega_{i j}{ }^{-}$is equal to the $-\omega_{i j}$, if $\omega_{i j}<0$ and is equal to zero otherwise. The term $e_{i j} \pm=s_{i}^{ \pm} s_{j}^{ \pm} / \nu^{ \pm}$stands for the expected density of positive or negative connection weights given a strength-preserved random null model, where $s_{i}^{ \pm}=\sum_{i}^{N} \omega_{i j}^{ \pm}$and $\nu^{ \pm}=$ $\sum_{i j}^{N} \omega_{i j}{ }^{ \pm}$. The Kronecker delta function $\delta_{M_{i}, M_{j}}$ is equal to one when the $i, j$ th nodes are within the same module and is equal to zero otherwise. We tested performance of a given separation of the networks into modules by applying community detection function community_louvian.m implemented in the Brain Connectivity Toolbox (version 201601-16; https://sites.google.com/site/bctnet/) while employing the vector of the node's affiliation with the particular lobe as initial community affiliation vector. 
We showed that the lobar organization of the cortical surface into frontal, parietal, temporal and occipital divisions is in fact modular (see Table B.2 and Fig. A.2. Accordingly, we then calculated the contribution of individual nodes to lobar modules as the node participation index and the within-module $z$-score, which we refer to as node between-lobes participation index and node within-lobe $z$-score.

Node between-lobes participation index: In general, the participation index $(p)$ assesses inter-modular connectivity. It is the ratio of within-lobe node edges to all other lobar modules in the network, where node $p_{i}$ tends to 0 if the node has links exclusively within its own module and tends to 1 if the node links exclusively outside of its own module. The weighted network participation is calculated by

$$
p_{i}^{w}=1-\sum_{m \in M}\left(\frac{k_{i}^{w}(m)}{k_{i}^{w}}\right)^{2},
$$

where $M$ is the set of modules and $k_{i}^{w}(m)$ is the weighted number of links of the $i$ th node to all other nodes in module $m$ - inter-modular degree and $k_{i}^{w}$ is the total degree of the $i$ th node. For the purpose of this study we will use term node 'between-lobes participation' for this network measure.

Node within-lobe degree $z$-score: The complement of the between lobes participation index is the normalized within-lobe degree $\left(z_{i}\right)$, which assess intra-lobar connectivity by means of $z$-scores, i.e. by the normalized deviation of the inter-lobar degree of a node from the respective mean degree distribution. Thus, node within-lobe $z$-score $\left(z_{i}\right)$ is large for a node with more intra-modular connections relative to the intermodular mean connectivity. For networks in which correlation strengths are preserved, the node withinmodule degree $z$-score is calculated in the following way:

$$
z_{i}^{w}=\frac{k_{i}^{w}\left(m_{i}\right)-\bar{k}^{w}\left(m_{i}\right)}{\sigma^{k^{w}}\left(m_{i}\right)}
$$

where $k_{i}^{w}(m)$ is as above and $\bar{k}_{i}^{w}\left(m_{i}\right)$ and $\sigma^{k^{w}}\left(m_{i}\right)$ are the mean and standard deviation of the within module $m_{i}$ degree distribution. Superscript $w$ indicates that these measures were calculated on weighted networks.

Node role in network modular organization: Node role with the modular lobar organization depends on its position in the $z-p$ parameter space. There are four possible roles that a node can have in the network (see for example Refs. 39 and 40), which are assigned on the basis of 'higher than average' measures of nodal properties. Here, we focus on only two of such roles, so called 'connectors' or global network hubs (that have high between-lobes participation and high within-lobe degree $z$-score) and so called 'provincial' hubs (that have high within-lobe degree $z$-score and low between-lobes participation). The threshold for high and low values of $z_{i}$ and $p_{i}$ are above 1.5 and 0.05 , respectively $\sqrt[39]{.}$

\subsection{Statistical analysis and visualization}

Statistical differences in demographic and cognitive scores were assessed using one-way analysis of variance (ANOVA) followed by post hoc $t$-tests of significant differences between the groups. The data were checked for normality of distribution using a onesample Kolmogorov-Smirnov test. The Chi-square test was used to test for differences in distribution of males and females between the groups. Statistical differences in global network correlation strength according to diagnostic groupings were tested using ANOVA for unbalanced sample size (to account for an uneven number of significant correlations across the networks). Node degree, within-lobe $z$-score $\left(z_{i}\right)$ and between-lobes participation index $\left(p_{i}\right)$ were compared across the groups using the Kruskal-Wallis test, a nonparametric ANOVA test. Results were reported as significant at the level $p<0.05$. Network visualization in brain space was performed using BrainNet. 41

\section{Results}

\subsection{Demographic, cognitive and gross morphological characteristics}

Table 1 shows demographic, cognitive and mean CT and SA for each group according to clinical diagnosis. The three groups differed significantly by age, $\mathrm{AD}$ patients being older than HE and bvFTD $\left(p<10^{-4}\right.$, in all tests). As expected, we also found significant differences in cognitive scores on the MMSE scale, $\mathrm{AD}$ patients being the most impaired and bvFTD more impaired than HE subjects $\left(p<10^{-4}\right.$ in all tests). The mean CT and total SA differed across groups. The differences between HE and both patient groups were significant in terms of both mean CT 
HE

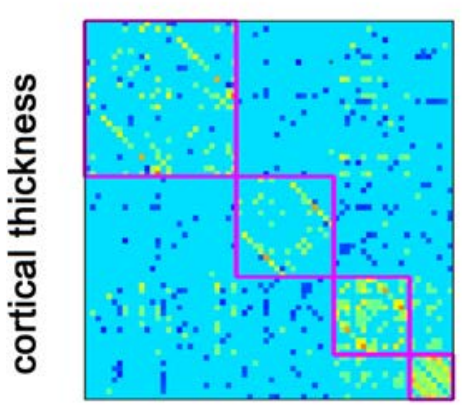

bvFTD

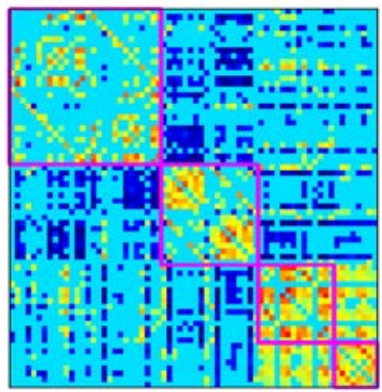

AD

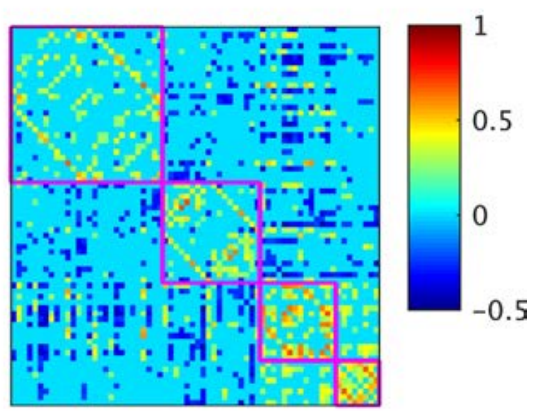

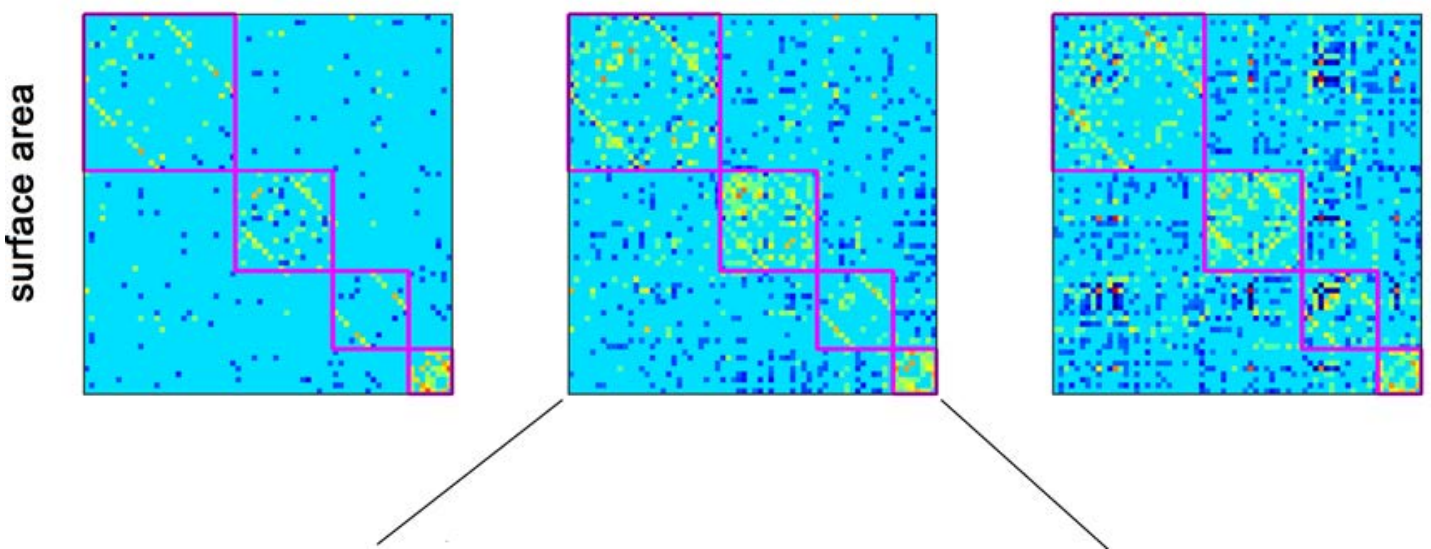

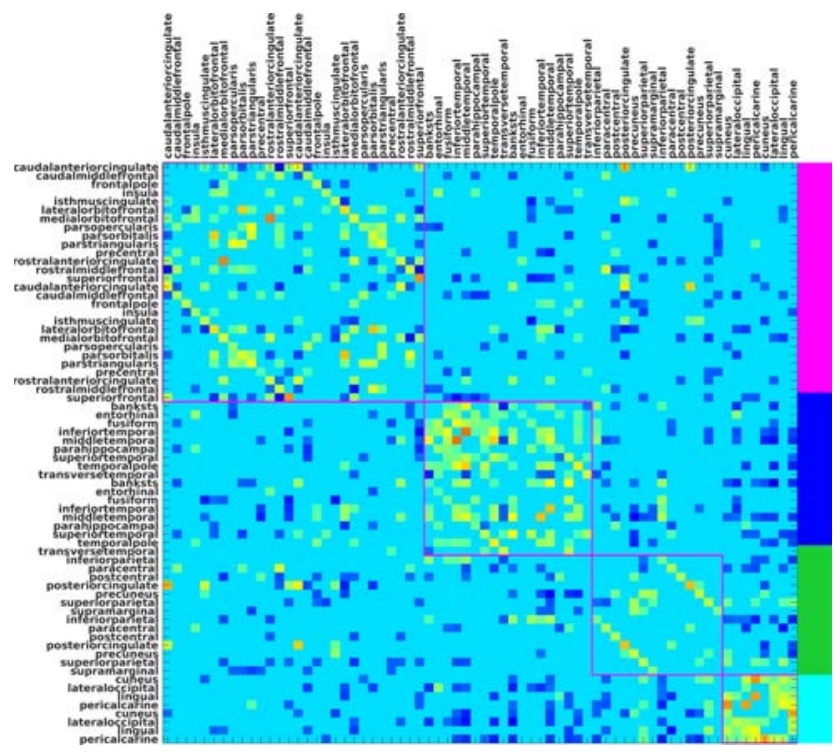

Fig. 1. (Color online) Group-based structural correlation networks depicted in the form of pair-wise correlation matrices: structural correlation matrix of cortical thickness (upper panel), structural correlation matrix of surface area (lower panel). The groups are: healthy elderly (HE), behavioral variant frontotemporal dementia (bvFTD) and Alzheimer's disease (AD). Each matrix element represents correlation strength (or edge strength) between 68 pairs of cortical surface areas from the Desikan-Killiany Atlas. Color bars to the right indicate correlation/edge strength. Sixty eight cortical surface regions (network nodes) are ordered according to their affiliations with the frontal, temporal, parietal and occipital lobes. Singlelobe regions are enclosed within squares and ordered from top to bottom/left to right: frontal, temporal, parietal and occipital. The enlarged matrix shows one group-based matrix, with the regional names displayed to the left and to the top of the matrix. The colored bar to the right of the matrix indicates node affiliation to the corresponding lobe (used throughout the paper): frontal (magenta), temporal (blue), parietal (green) and occipital (cyan). 
$\left(p<10^{-4}\right.$, in both tests) and total SA $(p<0.03$, in both tests) but bvFTD and AD groups did not differ from each other. The mean CT and total SA values averaged by brain lobes are provided in Table B.6 Thus, although AD and bvFTD differ in terms of lobar distribution of pathology, age and severity of cognitive impairment, neither overall extent of cortical thinning or changing mean surface area provide a means of distinguishing between the two conditions.

\subsection{Lobar properties of structural correlation network}

Since the definition of correlation-based network organization depends on the choice of the threshold value, $\stackrel{37}{,}$ we sought first to ensure that the networks we have defined are nonrandom in their global topology by calculating the density/sparsity value $(\kappa)$. Brain networks are considered to show nonrandom (small-world) topology if $\kappa \geq 0.1 .42$ We also ensured that inverse correlations were not omitted after thresholding (see Fig. A.1). Therefore, all positive and inverse CT and SA correlation networks considered in the present study are nonrandom. See also Table B.6 for global values of $\kappa$ for CT and SA in the three groups.

Using the modularity index, we sought to determine whether cortical lobes as conventionally defined correspond to network modules in the $\mathrm{CT} / \mathrm{SA}$ network. We found that only two homologue pairs (posterior cingulate and precentral cortex) in the CT network and two homologue pairs in the SA network [posterior cingulate cortex (PCC) and paracentral and right banstss (banks of the superior temporal sulcus)] were miss-assigned by the modularity index algorithm. Table B.2 gives details of the algorithm input and output. In practice, it is accepted that a $Q$ value above 0.3 is a good indicator of the existence of significant modules in a network ${ }^{43}$ To estimate the confidence interval of the $Q$ values for our data set we repeated calculations against $100 \mathrm{CT} / \mathrm{SA}$ matrices generated on corresponding surrogate data sets. Each of 100 surrogate CT and SA matrices was generated by randomly drawing 213 subjects from the three study groups and calculating $Q$ values on the correlation matrix obtained for CT and SA. The values of $Q$ are shown in Fig. A.2 for CT and SA networks. We can therefore conclude that the cortical lobes as conventionally described correspond to nonrandom modules in the CT/SA network.
The $68 \times 68$ matrices constructed from partial correlations for CT and SA nodes are shown according to diagnosis in Fig. 1. Nodes (i.e. cortical regions) in the matrix were ordered according to lobe. Color coding indicates the correlation strength or 'edge strength' between each pair of nodes. Zero entries (pale blue color in the matrix) correspond to nonsignificant correlations. We found that the significant network correlations had both positive and negative values (see Fig. A.1). Because of the number of significant inverse correlations and the apparent increase in correlation strength of the networks in bvFTD and $\mathrm{AD}$ relative to the $\mathrm{HE}$ group, we considered sub-networks of significant positive and negative correlations separately. Given the apparent differences in lobar network structure according to diagnostic group, we sought to determine whether these differences could be quantified.

\subsection{Mean correlation strength in $C T$ and $S A$ networks}

As shown in Figs. 2(a) and 2(b), the mean correlation strength for $\mathrm{CT}$ showed significant differences between HE, bvFTD and AD subjects in frontal, temporal, parietal and occipital lobes $\left(p<10^{-4}\right.$, for all tests). The mean correlation strength was higher in bvFTD and AD than in HE subjects in frontal, temporal, parietal and occipital lobes (for all pairwise comparisons $p \leq 0.003$ ). The mean correlation strength was higher in bvFTD than in AD in frontal $\left(p<10^{-4}\right)$ and temporal $(p=0.005)$ lobes.

The mean strength of networks of inverse correlations in the $\mathrm{CT}$ network also differed in frontal and temporal lobes [see Fig. 2(b)]. Again, both bvFTD and AD groups showed higher mean correlation strengths than the HE group in frontal and temporal lobes ( $p \leq 0.03$, in all tests), and the bvFTD group had higher mean inverse correlation strengths than $\mathrm{AD}$ group in frontal lobe $(p=0.003)$.

Figures 2(c) and 2(d)] shows significant differences in the mean correlation strength across the SA network. The diagnostic groups differed only in frontal lobe where the AD group had a lower mean correlation strength than the HE group $(p=0.03)$. Similarly, inverse SA network correlations differed significantly in the frontal lobe, with lower mean correlation strength in bvFTD and AD group when compared with the HE group ( $p \leq 0.04$, in both tests). This is due to a larger number of correlations 


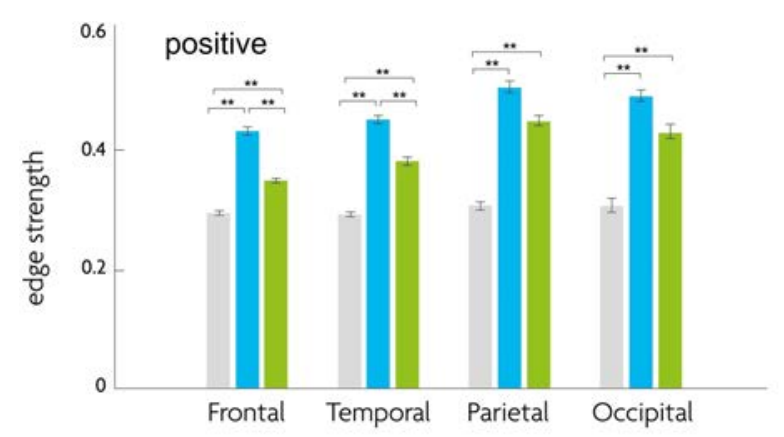

(a)

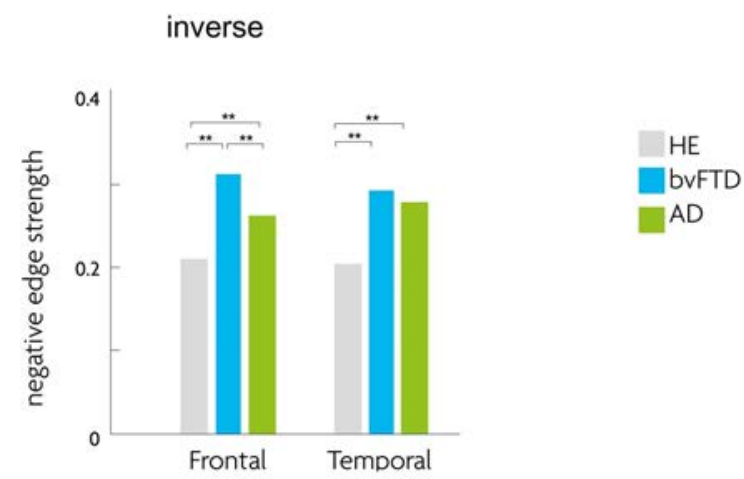

(c)

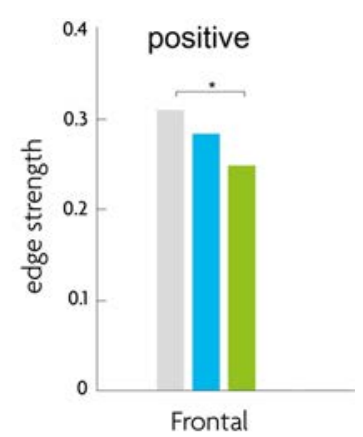

(b)

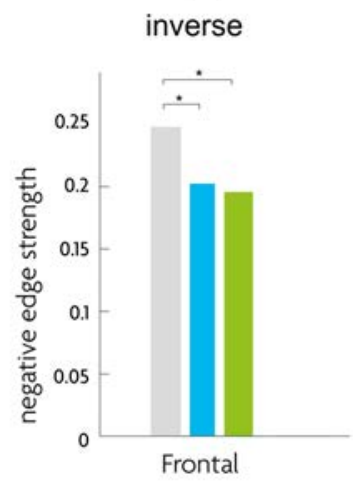

(d)

Fig. 2. Edge strength of cortical thickness ((a) and (b)) and surface area ((c) and (d)) correlation network averaged over brain lobes and compared between HE, bvFTD and AD groups. Data are shown for the networks of positive and inverse correlations (as indicated in the figure). Asterisks indicate significant differences between the three groups $\left({ }^{*} p<\right.$ $\left.0.05 ;{ }^{* *} p<0.01\right)$. Abbreviations: HE - healthy elderly, bvFTD - behavioral variant frontotemporal dementia, AD Alzheimer's disease.

with a broader frequency distribution in strength found in disease compared with sparser networks having a narrower frequency distribution in healthy elderly subjects (see Fig. A.1).

\subsection{Nodal measures of the CT network}

Node degree: Node degree, which quantifies the number of significant positive correlations per node is shown averaged over frontal, temporal, parietal and occipital lobes for the CT network in Fig. [3 (a) and 3(b)]. There were significant differences between groups in frontal, temporal, parietal and occipital lobes $\left(p<10^{-4}\right.$, in all tests). Both bvFTD and AD subjects had higher node degree in frontal and temporal lobes ( $p \leq 0.006$, for all tests) compared with HE subjects. The bvFTD group had notably higher node degree in parietal and occipital lobes than the $\mathrm{AD}$ group ( $p \leq 0.04$, for all tests). We found a similar pattern for the number of inverse correlations in the $\mathrm{CT}$ network in frontal, temporal, parietal and occipital lobes ( $p \leq 0.02$, in all tests). These differences were driven by a larger number of significant inverse correlations in bvFTD and AD than in the HE group across all four lobes $(p \leq 0.01$, in all tests). None of the differences between bvFTD and $\mathrm{AD}$ groups was significant.

Node between-lobes participation index: We found group differences in the node between-lobes participation index for CT. The index measures the extent of significant positive correlations with nodes in different lobes. This was significant for nodes located in the temporal, parietal, and occipital lobes $(p \leq 0.03$, in all tests). The differences reflect higher index values relative to the $\mathrm{HE}$ group in the parietal ( $p \leq 0.003$, in both groups), temporal $(p=0.01$, in $\mathrm{AD})$ and occipital $(p=0.002$, in bvfTD) lobes [see Fig. 3(c)]. None of the between-lobes participation index comparisons was significantly different in any lobe for the inverse correlations in the CT network. 


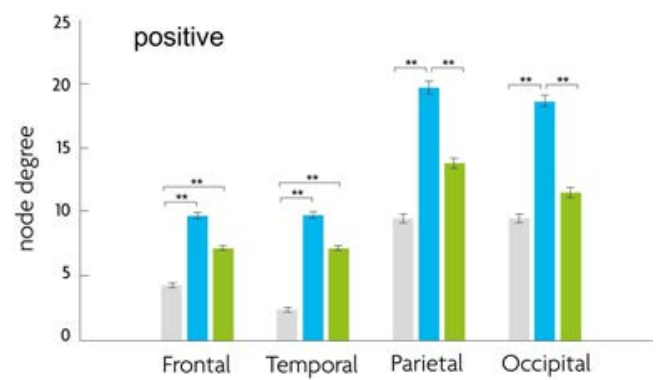

(a)

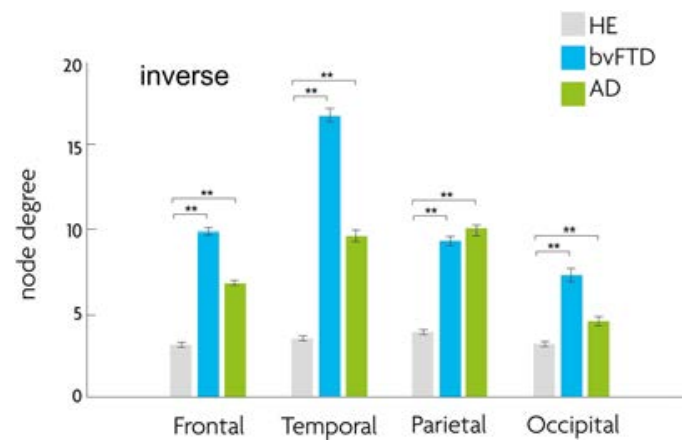

(b)

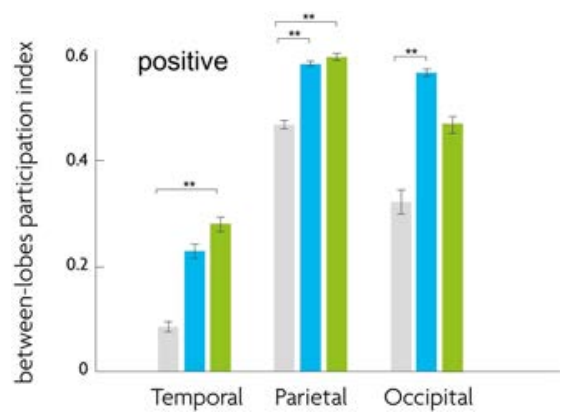

(c)

Fig. 3. Cortical thickness correlation network measures. Node degree ((a) and (b)) and between-lobes participation index (c) averaged over brain lobes and compared across HE, bvFTD and AD groups. Data are shown for the networks of positive and inverse correlations (as indicated in the figure). Asterisks indicate significant differences between the three groups $\left({ }^{*} p<0.05 ;{ }^{* *} p<0.01\right)$. Abbreviations: HE - healthy elderly, bvFTD - behavioral variant frontotemporal dementia, AD - Alzheimer's disease.

\subsection{Nodal measures in $S A$ network}

Node degree: Node degree values in SA network are shown for frontal, temporal, parietal and occipital lobes in Figs. [4(a) and 4(b)]. Positive correlations differed between diagnostic groups in frontal, temporal, parietal and occipital lobes $(p \leq 0.03)$. As with the CT network, both bvFTD and AD groups had higher SA node degree than the HE group in frontal, temporal and parietal lobes $\left(\left(p<10^{-4}\right.\right.$, in all tests). For the occipital lobe the only difference which was significant was between the $\mathrm{AD}$ and $\mathrm{HE}$ groups $(p=0.04)$. In contrast to the CT network, the node degree in parietal lobe was also significantly higher in AD than in bvFTD $(p=0.004)$.

The inverse correlation SA network also showed significant group differences in frontal, temporal, parietal and occipital lobes ( $p \leq 0.001$ in all tests). Again, both bvFTD and AD groups had higher node degree than the HE group in all four lobes $(p<0.001$ for all tests). In contrast to the CT inverse correlation network, the AD group had higher node degree than the bvFTD group in frontal $(p=0.02)$ and parietal $(p=0.01)$ lobes.

Node between-lobes participation index: Figures 4 (c) and 4 (d) show group differences in the nodal between-lobes participation index for the SA network organization. Both bvFTD and AD groups had higher index values than the HE group for the positive SA correlation network [Fig. 4(c)] in all four lobes $\left(p<10^{-4}\right)$. In contrast to the CT correlation network, the inverse SA correlation network [Fig. $4(d)]$ also showed significant differences in frontal and parietal lobes $(p \leq 0.04$ for both patients groups) and in temporal lobe for the $\mathrm{AD}$ group $(p \leq 0.001)$ relative to the HE group.

\subsection{Hubs of the structural correlation networks}

CT network hubs: There are four possible combinations of mean values of between-lobes participation index (high/low) and within-lobe $z$-score (high/low). We consider only the case of high or low between-lobes participation index and high withinlobe $z$-score in order to focus on nodes with hublike characteristics. Table B.3 provides data for the global and provincial network hubs. The number and distribution of network hubs with high $p_{i}$ and high $z_{i}$ values in the positive $\mathrm{CT}$ correlation network differed between study groups. In the HE subjects, hubs were distributed across the whole cortex such that each lobe had at least one hub, with four hubs in the frontal lobe. The re-organization of hub topology from HE to bvFTD and from HE to AD occurred differently in the two disease groups (see Fig. 5 (upper 


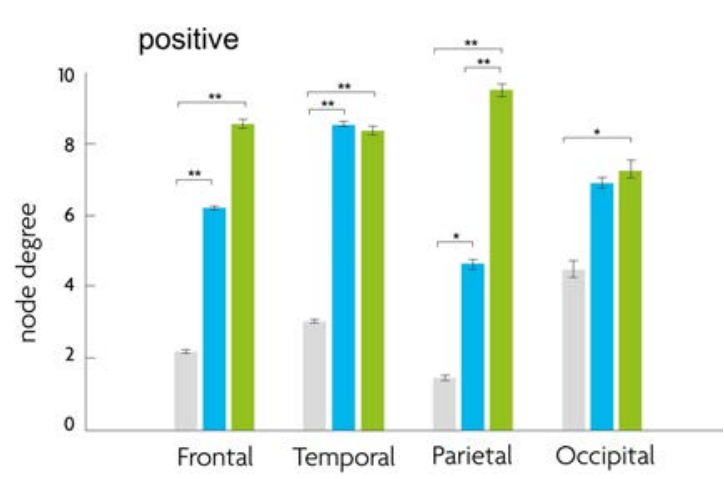

(a)

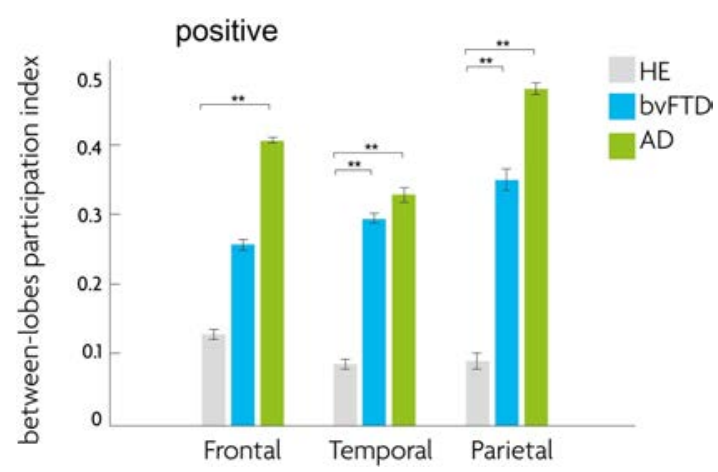

(c)

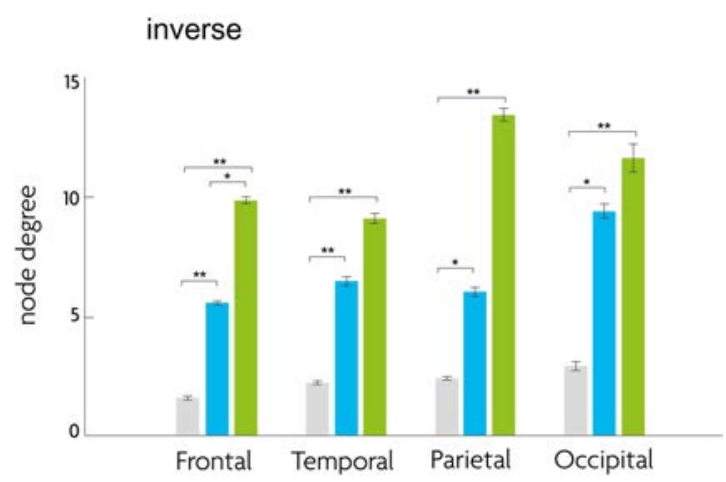

(b)

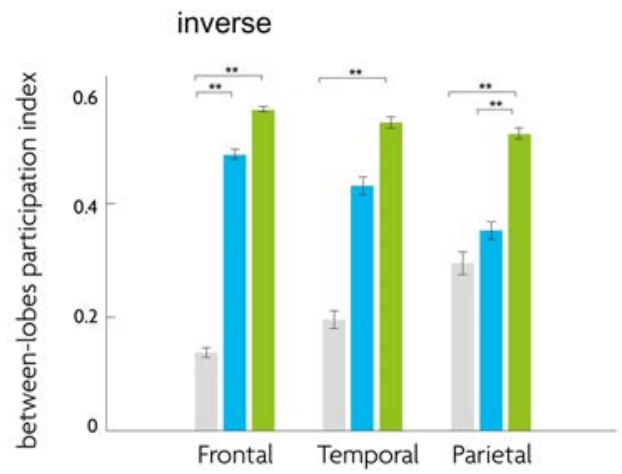

(d)

Fig. 4. Surface area correlation network measures. Node degree ((a) and (b)) and between-lobes participation index ((c) and (d)) averaged over brain lobes and compared across HE, bvFTD and AD groups. Data are shown for the networks of positive and inverse correlations (as indicated in the Figure). Asterisks indicate significant differences between the three groups $\left({ }^{*} p<0.05 ;{ }^{* *} p<0.01\right)$. Abbreviations: HE - healthy elderly, bvFTD - behavioral variant frontotemporal dementia, AD - Alzheimer's disease.

panel)). In bvFTD, the number of hubs in frontal lobe increased from 4 to 9 , decreased from 2 to 1 in the occipital lobe and vanished completely in parietal and occipital lobes. By contrast, hubs were distributed across all four lobes in $\mathrm{AD}$ almost equally. The number of hubs in the frontal lobe decreased (2 versus 4$)$, whereas the number increased relative to the $\mathrm{HE}$ group in temporal and occipital lobes (1 versus 3 and 2 versus 3 , respectively). A full list of nodes which have hub-like properties in the CT network is provided, along with lobar location, in Table B.3.

Nodes with hub-like properties in the negative correlation $\mathrm{CT}$ matrix were present exclusively in frontal and temporal lobes in all three groups, and their topological distribution differed between the groups(see Fig. 5 (lower panel) Table B.3).

SA network hubs: Hubs in the positive correlation SA network are shown in Fig. 6 Table B.4 provides a list of nodes and lobar locations classified according to between-lobe participation index and withinlobe $z$-score. A visual comparison of hub topology between groups shows that the left hemisphere had more nodes with hub-like properties in all diagnostic groups. However, HE subjects had only one SA hub (left insula), whereas both disease groups had more hubs in each lobe. The AD group had twice as many SA hubs compared to bvFTD (14 versus 7). Surprisingly, the bvFTD group had more SA hubs in the temporal than in the frontal lobe (4 versus 1$)$, while $\mathrm{AD}$ subjects had more frontal than temporal hubs (6 versus 4$)$. AD subjects had 3 hubs in the parietal lobe compared with 1 in bvFTD subjects.

Hubs in the negative correlation SA network were present in either frontal or temporal lobe only in all three groups. However, the HE group had one hub in the parietal lobe (precuneus) and bvFTD had two (inferiorparietal and paracentral) (see Table B.4). 

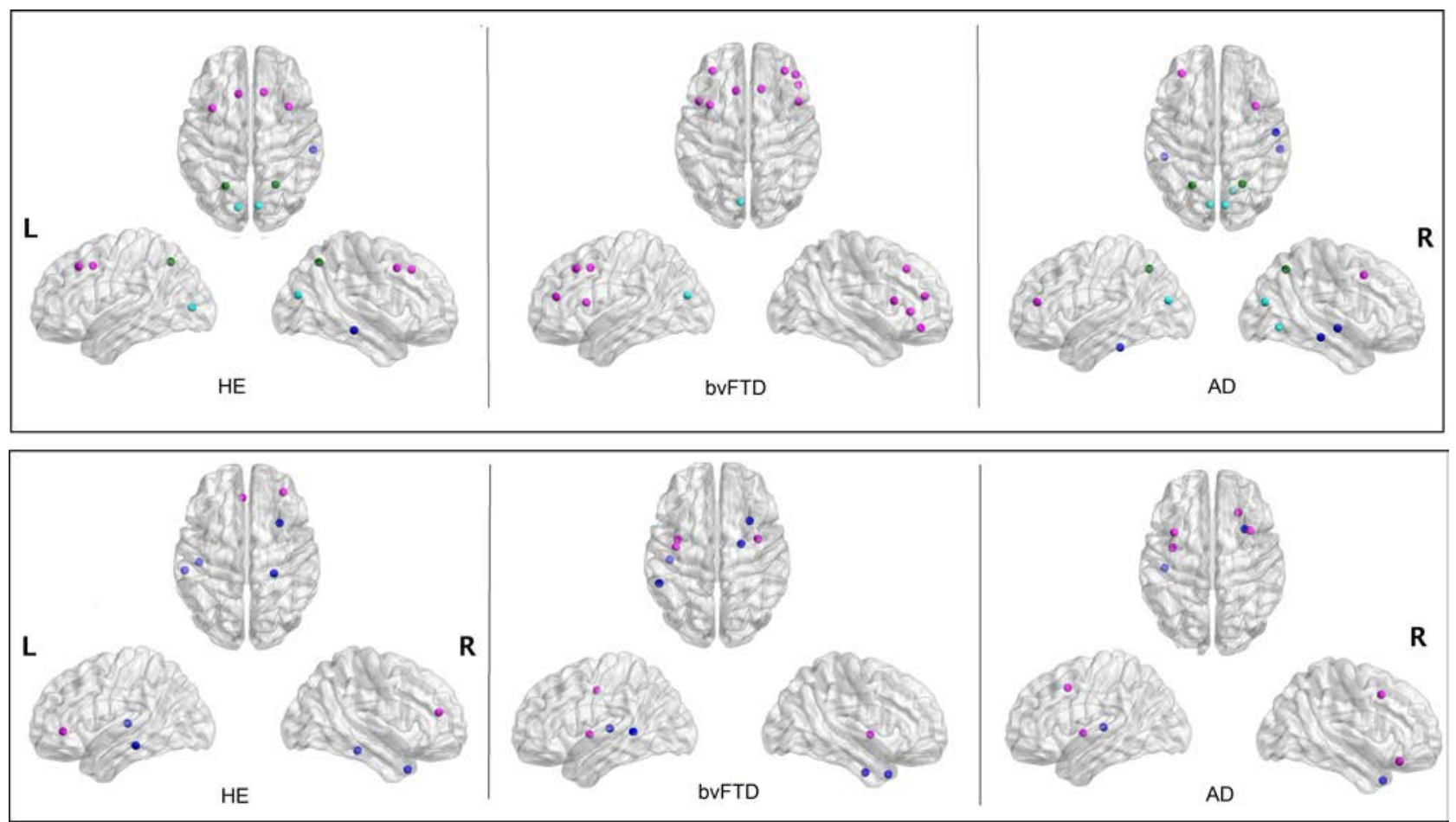

Fig. 5. (Color online) Hubs of the cortical thickness network visualized in brain space for positively (upper panel) and inversely (lower panel) correlated nodes. Colors indicate frontal (magenta), temporal (blue), parietal (green) or occipital (cyan) regions. Hubs are ranked according to high number of interactions between- and within-lobe. See Table B.3 for the list of regions/nodes. Abbreviations: HE - healthy elderly, bvFTD - behavioral variant frontotemporal dementia, AD Alzheimer's disease.

Interestingly, most of the inverse correlation SA hubs in $\mathrm{AD}$ were found in the frontal lobe.

\subsection{Cortical thickness - cortical surface area coupling topology}

The coupling strength between $\mathrm{CT}$ and SA nodes was calculated by element-wise multiplication of the corresponding $\mathrm{CT}$ and $\mathrm{SA}$ correlation matrices. Figure 7 shows $\mathrm{CT} / \mathrm{SA}$ coupling strength visualized in brain space. It can be seen that in HE subjects pairs of inter-hemispheric homologues show coupled CT/SA correlation. By contrast, $\mathrm{CT} / \mathrm{SA}$ coupling in $\mathrm{AD}$ and bvFTD groups are very similar to each other and different from the HE group. Both the bvFTD and $\mathrm{AD}$ groups showed more coupling between nonhomologous nodes in the same and contralateral hemispheres. The inter-lobar correlations (represented by grey lines) were also strikingly different between the bvFTD and AD groups. In the bvFTD group, most of the inter-lobar CT/SA correlations were due to fronto-temporal interactions. In $\mathrm{AD}$, most of the inter-lobar CT/SA coupling was due to frontoparietal interactions. A list of hubs of $\mathrm{CT} / \mathrm{SA}$ coupling topology is given in Table B.5

\section{Discussion}

We have examined structural correlation networks in subjects diagnosed clinically with either bvFTD or $\mathrm{AD}$ in three large global clinical trials, and compared these with healthy elderly subjects in a well-characterized birth cohort. For each group, networks were constructed from the partial correlations between $68 \times 68$ pairs of cortical surface areas (nodes) in terms of their thickness and surface area. The approach we have adopted has permitted a systematic analysis of both positive and inverse network correlations in the three clinical contexts. This study represents the first systematic comparative analysis of cortical thickness and surface area networks in a large population of subjects. Since the numbers needed to be comparable in the three groups, the overall study size was determined by the number of bvFTD subjects available. As this is a rare disease, 

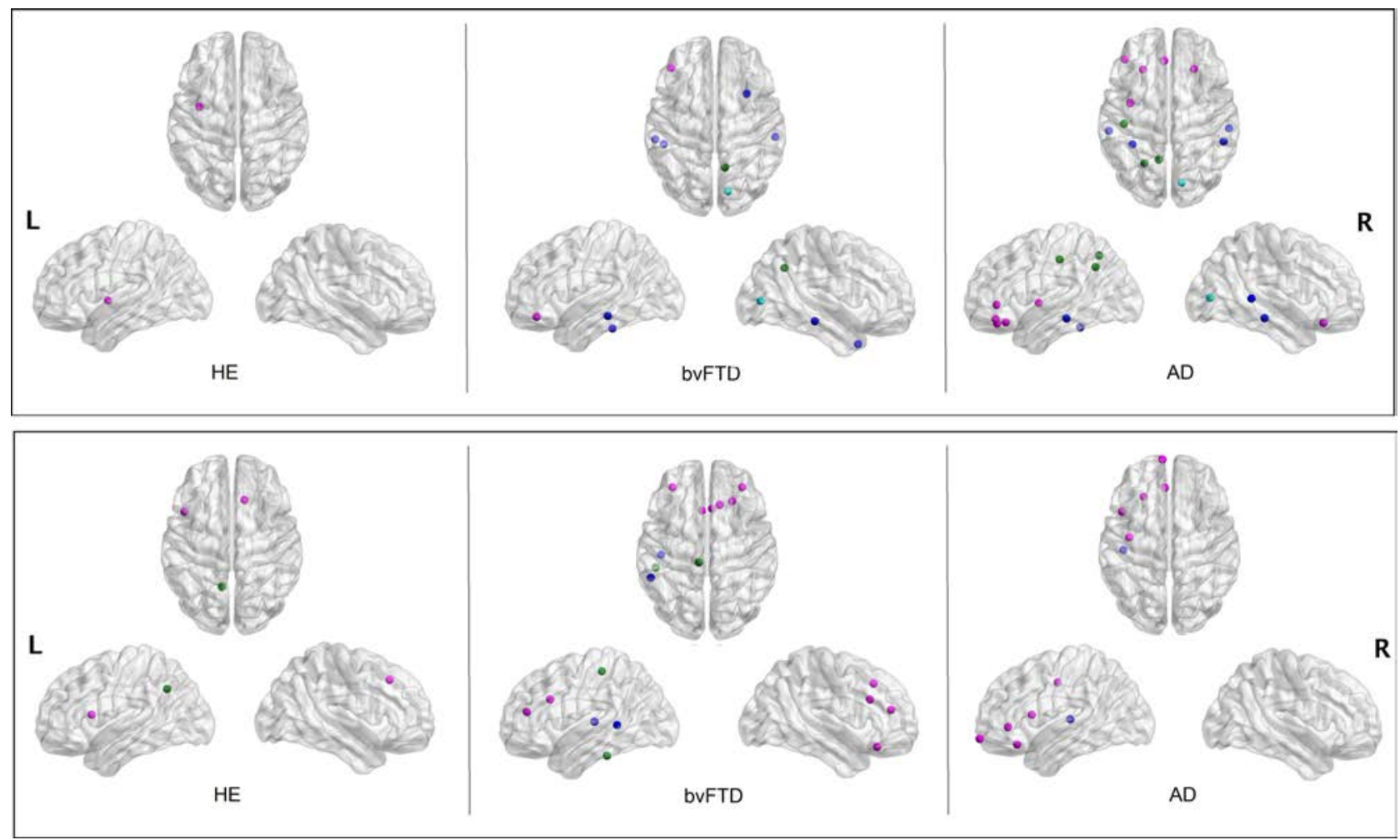

Fig. 6. (Color online) Hubs of the surface area network visualized in brain space for positively (upper panel) and inversely (lower panel) correlated nodes. Colors indicate frontal (magenta), temporal (blue), parietal (green) or occipital (cyan) regions. Hubs are ranked according to high number of interactions between- and within-lobe. See Table B.4 for the list of regions/nodes. Abbreviations: HE - healthy elderly, bvFTD - behavioral variant frontotemporal dementia, AD Alzheimer's disease.

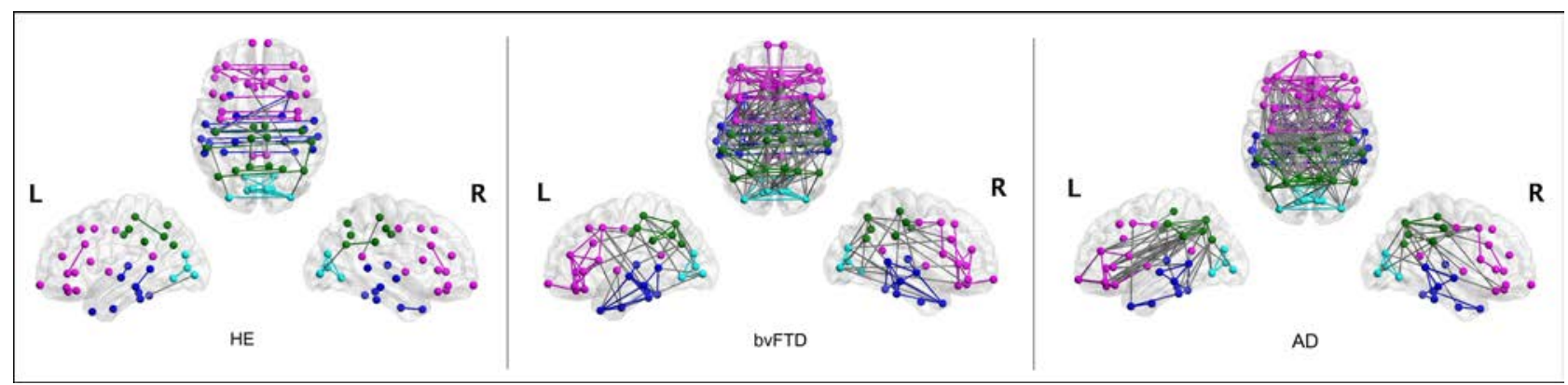

Fig. 7. (Color online) Interactions between cortical thickness and cortical surface area positive networks visualized in brain space. Colors indicate position within frontal (magenta), temporal (blue), parietal (green) or occipital (cyan) lobe. Node edges are colored according to the nodes they connect, within-lobe connections match nodes colors, between-lobes connections are grey. 
it was necessary for the bvFTD component of the study to be global, with patients entering from 70 trial sites in 13 countries. With 213 patients included in this study, this represents the largest set of MRI scan data in bvFTD subjects available to date. In order to match this, 213 patients were drawn randomly from a much larger group of $1132 \mathrm{AD}$ patients coming from 116 sites in 12 countries for study TRx237-005 and 128 sites in 16 countries for study TRx237-015. The 202 normal elderly subjects come from a well-characterized birth cohort that has been studied longitudinally ${ }^{[27}$ The findings we report are therefore robust and can be considered representative of international populations meeting accepted diagnostic criteria.

\subsection{Modularity of networks by lobes}

We first show that the structural correlations in frontal, temporal, parietal and occipital divisions of the cortical surface are inherently modular for both the cortical thickness and surface area networks. That is, our results confirm that the standard lobar divisions of the cortex share common network modularity attributes such that they differ from what would be expected in a comparable random network. Modules of highly clustered networks confer so-called 'small-world' properties and are thought to provide an optimal balance between local specialization and global integration. $\stackrel{44}{=}$ Our results from healthy elderly subjects are comparable with prior work in a smaller and younger healthy group revealing an underlying modular architecture in the regional thickness correlation network. ${ }^{45}$ We now report that this intrinsic lobe-wise modularity persists in both bvFTD and $\mathrm{AD}$, indicating that the overall lobar architecture of the networks is preserved in the presence of neurodegenerative change. As discussed further below, this contrasts with the hub-like organization of the networks which changes in a disease-specific manner.

\section{2. $\quad$ Similarities and differences between $A D$ and bvFTD relative to healthy elderly subjects}

This study represents first attempt to characterize the correlational networks in cortical thickness and surface area bvFTD and AD in a systematic manner in a large population. Although previous studies have examined structural integrity 46 and metabolic networks, ${ }^{9}$ these have been in substantially smaller numbers of subjects in a restricted research setting. It is therefore not known how representative the findings are more generally. In addition, although informative, the techniques used in these studies are much less available than routine MRI scanning. It would therefore be difficult to develop the earlier findings into an adjunctive diagnostic tool which could be used in routine clinical practice.

Both dementia patient groups were found to differ from healthy elderly subjects in highly significant ways. Both groups showed a striking increase in the overall correlation strength in thickness and surface area networks compared with healthy elderly subjects. The effect was more pronounced in the cortical thickness network in all lobes for both positive and negative correlations. This contrasts with a significantly lower correlation strength relative to normal for surface area in frontal lobe in AD and a directionally similar difference in bvFTD. This may be due to a larger number of correlations with a broader frequency distribution in disease compared to sparser networks having a narrower frequency distribution in healthy elderly subjects. In addition to increased overall correlation strength, the number of withinlobe positive and negative correlations as measured by node degree was higher in all lobes in both dementia groups than in healthy elderly controls. The number of between-lobe positive correlations in thickness as measured by the between-lobes participation index was also higher in all lobes. The number of within-lobe and between-lobe positive surface area correlations was also greater in both bvFTD and AD than in healthy elderly subjects in frontal, temporal and parietal lobes. Both disease groups also differed from healthy elderly subjects in terms of the correlations in coupling between cortical thickness and surface area. Thus, both diseases are characterized by an overall increase in the strength and extent of structural correlation, occurring both locally within lobes and globally between lobes.

The similarity between the two conditions in terms of the marked increase in overall strength and extent of structural correlation might appear to call into question the clinical distinctions between bvFTD and $\mathrm{AD}$ on which the classification of patients in this study was based. Indeed, there were no differences between the two conditions in terms of 
overall cortical thickness or surface area. However, there were a number of important network differences between the two conditions. In the cortical thickness network, the overall positive correlation strength was greater in bvFTD than in $\mathrm{AD}$ in frontal and temporal lobes, and the negative correlation strength was also greater in bvFTD than in AD in the frontal lobe. The number of significant positive within-lobe correlations was higher in bvFTD than in $\mathrm{AD}$ in parietal and occipital lobes. Conversely, the number of both positive and negative withinlobe correlations was greater in $\mathrm{AD}$ than in bvFTD in frontal and parietal lobes. Most of the inverse correlations in cortical thickness and surface area related to inter-hemispheric nonhomologous frontotemporal lobes in bvFTD and in fronto-parietal lobes in $\mathrm{AD}$.

The hub-like organization of the correlation networks also differed substantially in the two conditions. Network connector hubs are thought to provide network integration, whilst provincial hubs provide network segregation. It has been proposed that hubs provide resilience to insult in neurodegenerative disorders ${ }^{40}$ Alternatively, it has been suggested that hubs represent loci of particular vulnerability $\underline{47}$ It is therefore of interest to study how hubs change in the context of neurodegenerative disease. bvFTD is characterized by an increase in the number of cortical thickness hubs in frontal lobe and a reduction or elimination of hubs in temporal, parietal and occipital lobes. By contrast, AD is characterized by hubs distributed in all lobes, a reduction in the number of hubs in frontal cortex, and an increase in hubs in temporal and occipital lobes compared with bvFTD. In the positive correlation network for surface area, $A D$ subjects had twice as many hubs overall than bvFTD, and the topology of these hubs differed. Thus overall, AD is characterized by a much more distributed pattern of hubs in both the thickness and surface area networks than bvFTD. By contrast, the hub-like organization is much more localized in bvFTD. It has been argued that bvFTD is clinical syndrome with focal but heterogeneous atrophy centered around hubs $\frac{48}{4 d e n t i f i c a t i o n ~ o f ~ t h e ~ i n s u-~}$ lar region as one of the inverse network hubs (in both bvFTD and AD groups for the CT network) is consistent with the recent unexpected finding from diffusion MRI that there is an increase in hub-like fiber connectivity of the insula fined in bvFTD 49
The hubs of the healthy elderly group, on the other hand, are highly connected within and between lobes in a homologous fashion and are not otherwise linked to each other. The differences in hub-like organization between $\mathrm{AD}$ and bvFTD indicate differences in the hierarchy of nodal vulnerability and in the organization of network adaptation compensating differently in the two conditions. Thus, unlike lobar modularity, which is preserved in neurodegenerative disease, a constant hub-like organization is not preserved, implying that pre-existing hubs are not an intrinsic structural property of cortical network organization.

Although $\mathrm{AD}$ is also characterized by changes in cortical thickness, these are on the whole less marked than in bvFTD, whereas the changes in surface area are more prominent in $\mathrm{AD}$, suggesting coordinated changes in numbers of adjacent affected columns. These differences would be consistent with the pathology of bvFTD affecting interneurons and astrocytes which have more localized links. The predominance of surface area correlations in $\mathrm{AD}$ would be consistent with the pathology affecting primarily long-tract cortico-cortical projection systems mediated by the principal cells. bvFTD differs in a number of important respects from $\mathrm{AD}$ : there is no cholinergic deficit in bvFTD, 50 there is no treatment benefit from treatment with either acetylcholinesterase inhibitors 51 or memantine $\sqrt[52]{52}$ bvFTD is characterized by prominent astrocytic pathology, 53 neurons affected in neocortex are predominantly spiny interneurons in layers II and VI (pyramidal cells in layers III and V predominantly affected in $\mathrm{AD}^{544}$ ) and dentate gyrus of hippocompus (neurons affected in $\mathrm{AD}$ are in $\mathrm{CA} 1-4$ and not dentate gyrus $\sqrt{55156}$ and bvFTD is characterized by increased glutamate levels in neocortex but $\mathrm{AD}$ is not ${ }^{57}$ However, none of these considerations provides a simple explanation for the different distribution patterns of the correlated structural changes we have described.

\subsection{Global character and significance of network changes in dementia}

The overall picture that emerges from the two disease groups is that network architecture is changed in a coordinated fashion throughout the whole brain as regards both positive and inverse correlations. 
This is surprising, given that the neurodegenerative processes in these two conditions are generally considered to be anatomically restricted, to frontal and temporal lobes in the case of bvFTD, and to temporal and parietal lobes in AD. Rather, the network analysis suggests that there are changes in cortical thickness and surface area networks in both conditions that affect all lobes in a global manner, but that there are differences in the anatomical topology of the changes. Both Tau and TDP-43 aggregation pathology is known to spread in prion-like fashion, whereby pathology in an affected neuronal population can initiate pathology in a connected, but previously unaffected neuronal population. $\frac{58159}{}$ The positive correlations could therefore reflect in part the spread of pathology in existing normal networks whereby existing functional networks are affected or spared together. Alternatively, such correlations might express functional dependencies, such that loss of function in one member of a partnership results is a parallel loss of function in a partner normally synchronized functionally with an affected node. This interpretation would be consistent with previous work on cortical thickness correlations in healthy adults, $\frac{13[60}{16}$ where positive correlations have been found to converge with diffusion-based axonal connections. $\underline{60}$

The present study has highlighted for the first time the significance of inverse correlational networks. It should be noted that because the inverse correlations seen in both neurodegenerative disorders reflect primarily inter-lobar nonhomologous associations, they would not have been detected using only a lobe-based approach to the analysis. It is particularly the appearance of these nonhomologous inverse inter-lobar correlations and their increased strength that represents the clearest overall difference between neurodegenerative disease and normal aging. By contrast, the normal aging brain is characterized by substantially weaker homologous positive correlations. An attractive hypothesis is that as certain nodes become functionally impaired, other still unaffected nodes compensate, accentuating nonhomologous associations in disease, as also suggested in some fMRI studies in $\mathrm{AD} \cdot 61$ This would imply that the major reorganization in structural networks that we have documented may be partly adaptive in character. Structural plasticity has been demonstrated in other contexts, $\frac{62}{2}$ and functional compensation is known to occur in focal disease ${ }^{63}$ We are currently investigating whether and how the structural adaptations we describe relate to differential interactions between default mode and salience networks in $\mathrm{AD}$ and bvFTD ${ }^{64}$ We are also investigating the extent to which symptomatic and disease-modifying treatments differ in their impact on the structural networks we have described. If the changes are adaptive, it would be expected that symptomatic treatments would accentuate compensatory network changes, whereas a disease-modifying treatment would be expected to reduce the strength of compensatory network adaptations by normalizing function in neuronal populations affected by pathology.

\subsection{Study limitations}

The main limitation of the present study is that, because the matrices we have used to define networks depend on correlations across subjects, we have no means of analyzing how network changes in individuals relate to changes in cognitive and functional capacity. The group-wise approach we have used provides a foundation for further work aimed at understanding disease-specific variability at the individual level. Another potential limitation is that the study is based on the anatomical parcellation into 68 cortical regions provided by the DesikanKilliany Atlas. ${ }^{32}$ Although well established, there are also well defined alternatives. Further work is required to understand how different anatomical parcellations could affect patterns of network interaction and their interpretation. Another important limitation is that direct measurements of anatomical tracts from diffusion-weighted images can provide better information about changes in axonal connectivity than statistical dependences in regional morphological measures presented in this study. However, our results are comparable with anatomical connectivity networks defined using this technique $\underline{\underline{60}}$ This limitation could be addressed in future work combining diffusion- and T1-weighted MR images in similar settings, or combining T1 MRI data with EEG approaches which map strength and direction of nodal connections $\frac{65}{65}$ However, the countervailing advantage of the approach we have adopted is that it makes use of standard MRI scans that are widely available clinically, and could therefore provide a 
basis for development of analytic approaches which could be useful in routine clinical practice.

\subsection{Conclusions}

This work represents the first comprehensive comparative study of correlated structural network abnormalities in bvFTD and AD relative to healthy aging. These correlations arise from both positively and inversely linked changes in cortical thickness and surface area in the two disease conditions, which are quite different from those seen in normal elderly subjects. The changes seen in disease are global in character and are not restricted to fronto-temporal and temporo-parietal lobes, respectively, in bvFTD and AD. Rather, they appear to represent structural adaptations to neurodegeneration which differ in the two conditions. All of the correlational networks show a quite distinctive hub-like organization which differs both from normal and between the two forms of dementia. Unlike lobar organization of networks, which remains constant in disease, hublike organization varies with the underlying pathology. This implies that hub-like organization is not a fixed feature of the brain and attempts to explain disease in terms of hubs may be inadequate. The differences between $\mathrm{AD}$ and bvFTD that we have documented confirm that the clinical differences in the two dementia populations correspond to systematic differences in the underlying network structure of the cortex. The topological differences in thickness and surface-area hub-like organization, as well as the underlying positive and negative correlation networks, may provide a basis for development of analytical tools to aid in the differential diagnosis in the two conditions which can be difficult to distinguish by purely clinical criteria.

\section{Acknowledgments}

We would like to acknowledge the support of the Maxwell compute cluster funded by the University of Aberdeen. We also gratefully acknowledge study investigators and the generosity of study participants.

\section{Appendix A. Construction of Structural Networks}

Group-wise structural networks were constructed on partial correlations between 68 regional thickness/surface area of the Desikan-Killiany Atlas .32 We retained all significant correlations (either being positive or negative) and considered positive and negative sub-networks separately when calculating network attributes.
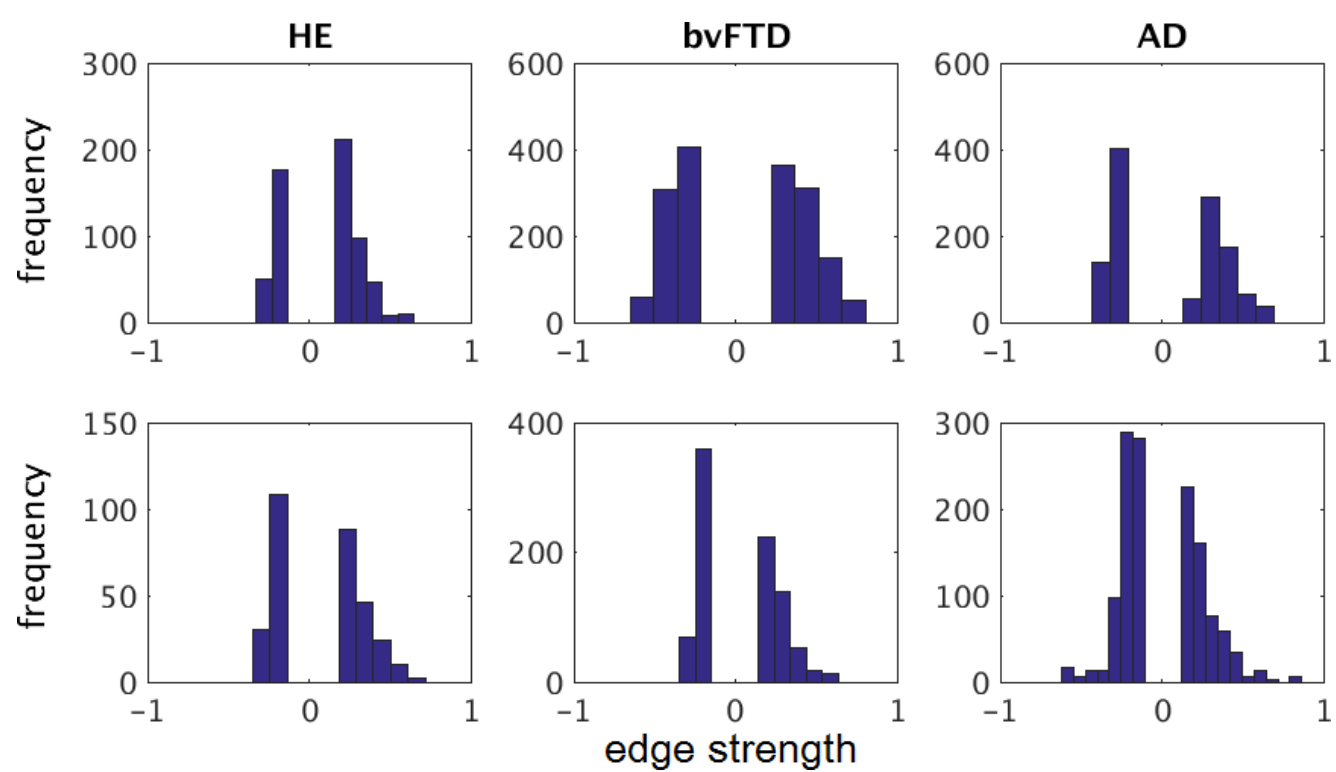

Fig. A.1. Histograms of the retained edges in cortical thickness (upper panel) and surface area (lower panel) correlation network. Retained edges contain both positive and negative correlations. Abbreviations: HE — healthy elderly, bvFTD — behavioral variant frontotemporal dementia, AD — Alzheimer's disease. 


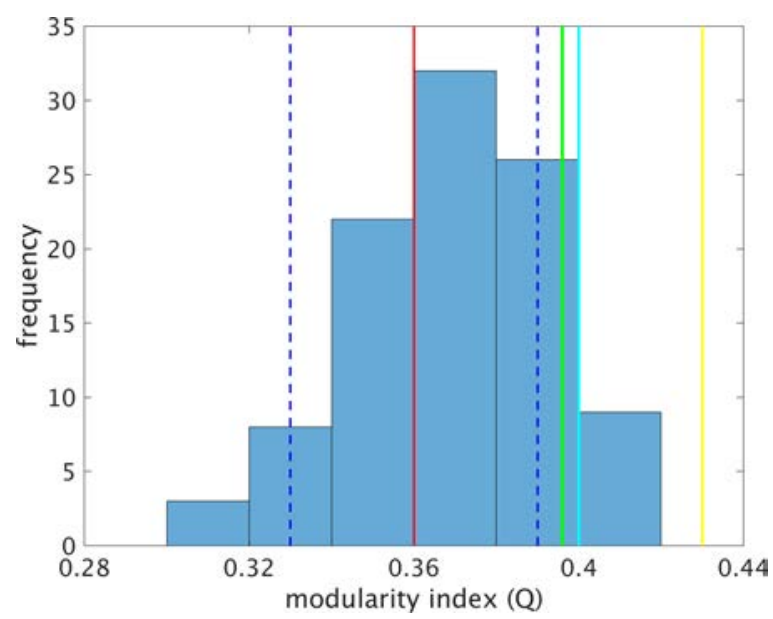

(a)

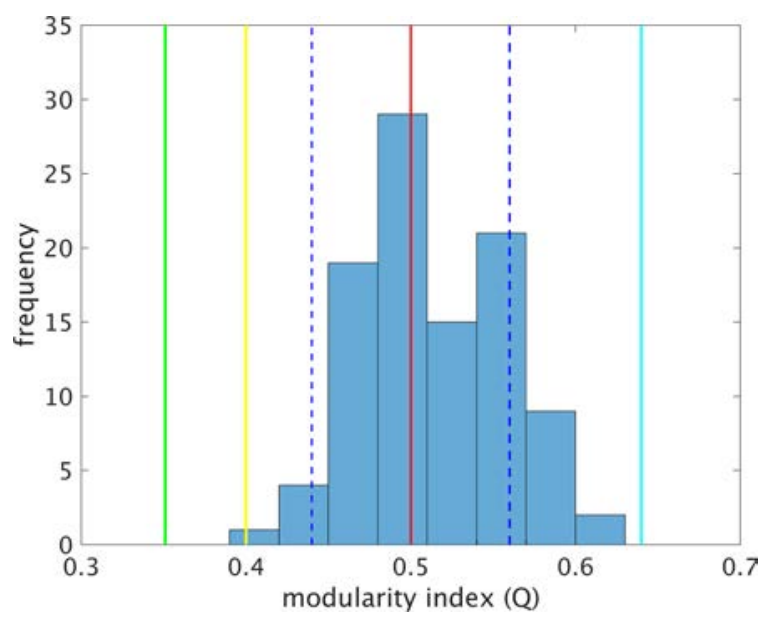

(b)

Fig. A.2. (Color online) Distribution of the modularity index $(Q)$ in cortical thickness $(A)$ and surface area $(B)$ networks generated on 100 surrogate data sets. Each surrogate CT/SA correlation matrix was constructed on partial correlations between regional CT/SA across 213 participants randomly drawn from 628 participants of the three study groups. Red line indicates mean value and blue dashed lines indicate 1.5 standard deviations away from the mean $\left(Q_{\mathrm{CT}}=0.36 \pm 0.02\right)$ and $\left(Q_{\mathrm{SA}}=0.50 \pm 0.06\right)$, i.e. a range where $Q$ values are similar to that of a random graph. For the study groups we obtained the following values: $Q_{\mathrm{HE}}=0.396$ (green), $Q_{\mathrm{bvFTD}}=0.43$ (yellow) and $Q_{\mathrm{AD}}=0.40$ (cyan) for CT network and $Q_{\mathrm{HE}}=0.35$ (green), $Q_{\mathrm{bvFTD}}=0.40$ (yellow) and $Q_{\mathrm{AD}}=0.64$ (cyan) for SA network.
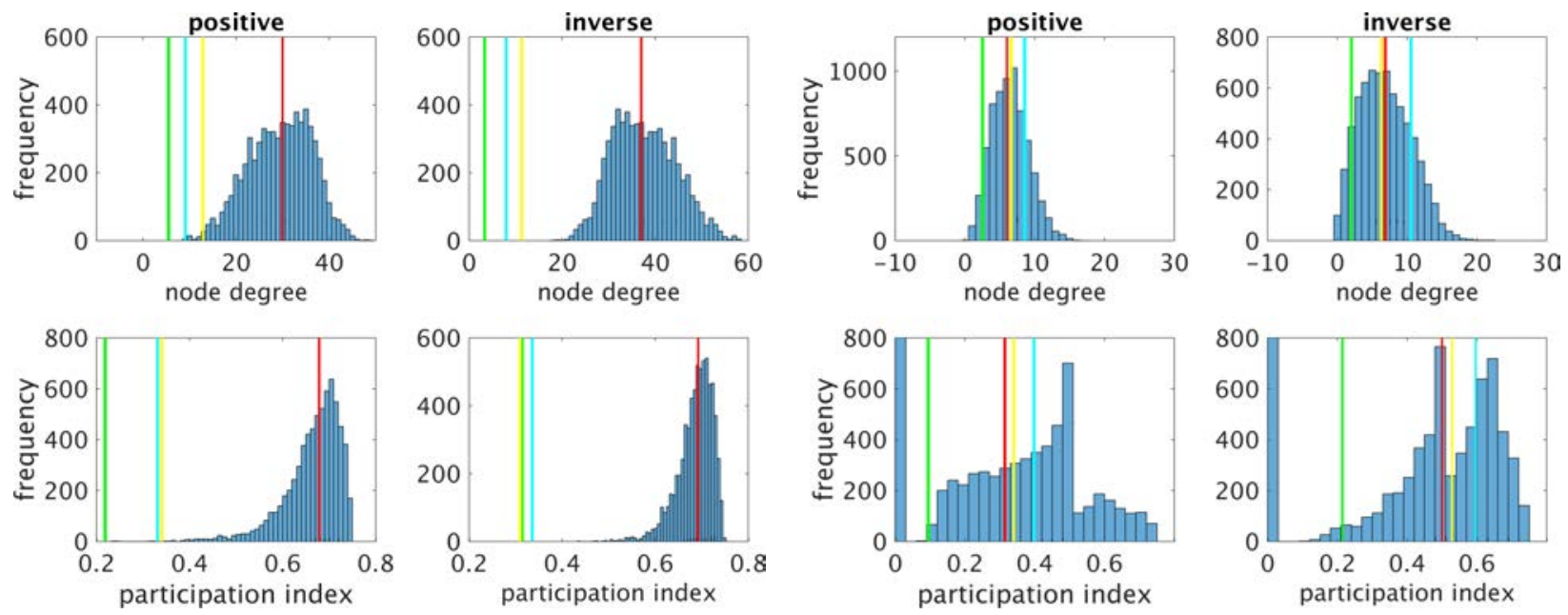

(a)

(b)

Fig. A.3. (Color line) Distribution of node degree and participation index calculated for cortical thickness (a) and surface area (b) network of the surrogate data sets. Solid red lines indicate mean (node degree) or median (participation index) values for surrogates. Green, yellow and cyan indicate corresponding values for HE, bvFTD or AD group respectively. 

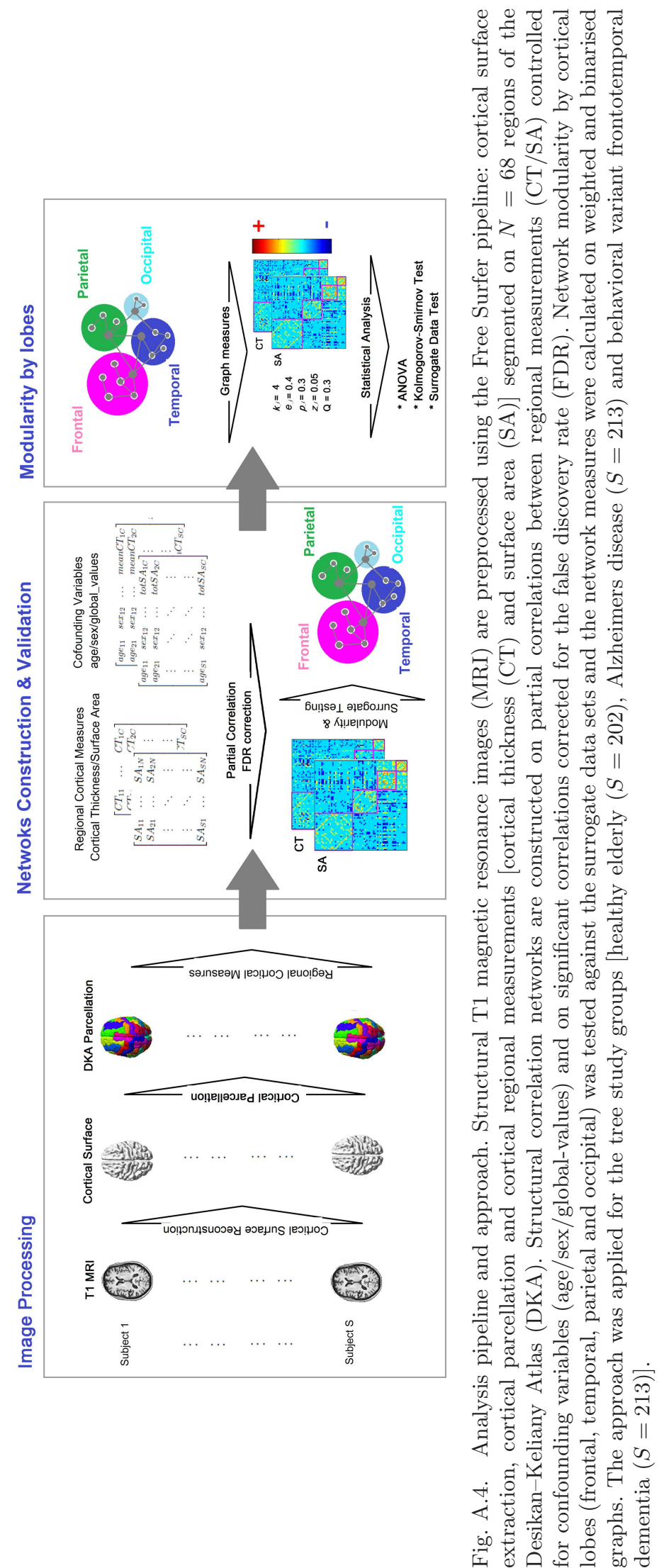


\section{Appendix B. Lists of Cortical Regions and Structural Networks Hubs}

Table B.1. Cortical surface of the frontal, temporal, parietal or occipital lobe areas according to the Desikan-Killiany (DK) Atlas. Cortical regions (nodes) of the each structural correlation matrix are ordered according to the list below throughout the study.

\begin{tabular}{lc}
\hline Region label & Lobe \\
\hline Caudal anterior cingulate & $\mathrm{F}$ \\
Caudal middle frontal & $\mathrm{F}$ \\
Frontal pole & $\mathrm{F}$ \\
Insula & $\mathrm{F}$ \\
Isthmus cingulate & $\mathrm{F}$ \\
Lateral orbitofrontal & $\mathrm{F}$ \\
Medial orbitofrontal & $\mathrm{F}$ \\
Parsopercularis & $\mathrm{F}$ \\
Parsorbitalis & $\mathrm{F}$ \\
Parstriangularis & $\mathrm{F}$ \\
Precentral & $\mathrm{F}$ \\
Rostral anterior cingulate & $\mathrm{F}$ \\
Rostral middle frontal & $\mathrm{F}$ \\
Superior frontal & $\mathrm{F}$ \\
Banksts & $\mathrm{T}$ \\
Entorhinal & $\mathrm{T}$ \\
Fusiform & $\mathrm{T}$ \\
Inferior temporal & $\mathrm{T}$ \\
Middle temporal & $\mathrm{T}$ \\
Parahippocampal & $\mathrm{T}$ \\
Superior temporal & $\mathrm{T}$ \\
Temporal pole & $\mathrm{T}$ \\
Transverse temporal & $\mathrm{T}$ \\
Paracentral & $\mathrm{P}$ \\
Postcentral & $\mathrm{P}$ \\
Posterior cingulate & $\mathrm{P}$ \\
Srecuneus & $\mathrm{P}$ \\
Suprior parietal & $\mathrm{P}$ \\
Cuneus & $\mathrm{P}$ \\
Lateral occipital & $\mathrm{P}$ \\
Pingual & $\mathrm{O}$ \\
\hline & $\mathrm{O}$ \\
\hline
\end{tabular}

Table B.2. Node assignment to the frontal, temporal, parietal of occipital lobe by the algorithm and by the Desikan-Killiany Atlas (DKA) cortical parcellation. Red are nodes wrongly assigned to a lobe for cortical thickness (CT) network and blue, are those incorrectly assigned for surface area (SA) network.

\begin{tabular}{|c|c|c|c|c|c|c|c|c|}
\hline \multirow{3}{*}{$\frac{\text { Region }}{\text { Banksts }}$} & \multicolumn{4}{|c|}{ CT network } & \multicolumn{4}{|c|}{ SA network } \\
\hline & \multicolumn{2}{|c|}{$\begin{array}{l}\text { Label } \\
\text { by } \\
\text { algo- } \\
\text { rithm }\end{array}$} & \multicolumn{2}{|c|}{$\begin{array}{l}\text { Label } \\
\text { by } \\
\text { DKA }\end{array}$} & \multicolumn{2}{|c|}{$\begin{array}{l}\text { Label } \\
\text { by } \\
\text { algo- } \\
\text { rithm }\end{array}$} & \multicolumn{2}{|c|}{$\begin{array}{l}\text { Label } \\
\text { by } \\
\text { DKA }\end{array}$} \\
\hline & $\mathrm{T}$ & $\mathrm{T}$ & $\mathrm{T}$ & $\mathrm{T}$ & $\mathrm{T}$ & $\mathrm{P}$ & $\mathrm{T}$ & $\mathrm{T}$ \\
\hline $\begin{array}{c}\text { Caudal anterior } \\
\text { cingulate }\end{array}$ & $\mathrm{F}$ & $\mathrm{F}$ & $\mathrm{F}$ & $\mathrm{F}$ & $\mathrm{F}$ & $\mathrm{F}$ & $\mathrm{F}$ & $\mathrm{F}$ \\
\hline Caudal middle frontal & $\mathrm{F}$ & $\mathrm{F}$ & $\mathrm{F}$ & $\mathrm{F}$ & $\mathrm{F}$ & $\mathrm{F}$ & $\mathrm{F}$ & $\mathrm{F}$ \\
\hline Cuneus & $\mathrm{O}$ & $\mathrm{O}$ & $\mathrm{O}$ & $\mathrm{O}$ & $\mathrm{O}$ & $\mathrm{O}$ & $\mathrm{O}$ & $\mathrm{O}$ \\
\hline Entorhinal & $\mathrm{T}$ & $\mathrm{T}$ & $\mathrm{T}$ & $\mathrm{T}$ & $\mathrm{T}$ & $\mathrm{T}$ & $\mathrm{T}$ & $\mathrm{T}$ \\
\hline Frontal pole & $\mathrm{F}$ & $\mathrm{F}$ & $\mathrm{F}$ & $\mathrm{F}$ & $\mathrm{F}$ & $\mathrm{F}$ & $\mathrm{F}$ & $\mathrm{F}$ \\
\hline Fusiform & $\mathrm{T}$ & $\mathrm{T}$ & $\mathrm{T}$ & $\mathrm{T}$ & $\mathrm{T}$ & $\mathrm{T}$ & $\mathrm{T}$ & $\mathrm{T}$ \\
\hline Inferior parietal & $\mathrm{P}$ & $\mathrm{P}$ & $\mathrm{P}$ & $\mathrm{P}$ & $\mathrm{P}$ & $\mathrm{P}$ & $\mathrm{P}$ & $\mathrm{P}$ \\
\hline Inferior temporal & $\mathrm{T}$ & $\mathrm{T}$ & $\mathrm{T}$ & $\mathrm{T}$ & $\mathrm{T}$ & $\mathrm{T}$ & $\mathrm{T}$ & $\mathrm{T}$ \\
\hline Insula & $\mathrm{F}$ & $\mathrm{F}$ & $\mathrm{F}$ & $\mathrm{F}$ & $\mathrm{F}$ & $\mathrm{F}$ & $\mathrm{F}$ & $\mathrm{F}$ \\
\hline Isthmus cingulate & $\mathrm{F}$ & $\mathrm{F}$ & $\mathrm{F}$ & $\mathrm{F}$ & $\mathrm{F}$ & $\mathrm{F}$ & $\mathrm{F}$ & $\mathrm{F}$ \\
\hline Lateral occipital & $\mathrm{O}$ & $\mathrm{O}$ & $\mathrm{O}$ & $\mathrm{O}$ & $\mathrm{O}$ & $\mathrm{O}$ & $\mathrm{O}$ & $\mathrm{O}$ \\
\hline Lateral orbitofrontal & $\mathrm{F}$ & $\mathrm{F}$ & $\mathrm{F}$ & $\mathrm{F}$ & $\mathrm{F}$ & $\mathrm{F}$ & $\mathrm{F}$ & $\mathrm{F}$ \\
\hline Lingual & $\mathrm{O}$ & $\mathrm{O}$ & $\mathrm{O}$ & $\mathrm{O}$ & $\mathrm{O}$ & $\mathrm{O}$ & $\mathrm{O}$ & $\mathrm{O}$ \\
\hline Medial orbito frontal & $\mathrm{F}$ & $\mathrm{F}$ & $\mathrm{F}$ & $\mathrm{F}$ & $\mathrm{F}$ & $\mathrm{F}$ & $\mathrm{F}$ & $\mathrm{F}$ \\
\hline Middle temporal & $\mathrm{T}$ & $\mathrm{T}$ & $\mathrm{T}$ & $\mathrm{T}$ & $\mathrm{T}$ & $\mathrm{T}$ & $\mathrm{T}$ & $\mathrm{T}$ \\
\hline Paracentral & $\mathrm{P}$ & $\mathrm{P}$ & $\mathrm{P}$ & $\mathrm{P}$ & $\mathrm{F}$ & $\mathrm{F}$ & $\mathrm{P}$ & $\mathrm{P}$ \\
\hline Parahippocampal & $\mathrm{T}$ & $\mathrm{T}$ & $\mathrm{T}$ & $\mathrm{T}$ & $\mathrm{T}$ & $\mathrm{T}$ & $\mathrm{T}$ & $\mathrm{T}$ \\
\hline Pars opercularis & $\mathrm{F}$ & $\mathrm{F}$ & $\mathrm{F}$ & $\mathrm{F}$ & $\mathrm{F}$ & $\mathrm{F}$ & $\mathrm{F}$ & $\mathrm{F}$ \\
\hline Pars orbitalis & $\mathrm{F}$ & $\mathrm{F}$ & $\mathrm{F}$ & $\mathrm{F}$ & $\mathrm{F}$ & $\mathrm{F}$ & $\mathrm{F}$ & $\mathrm{F}$ \\
\hline Pars triangularis & $\mathrm{F}$ & $\mathrm{F}$ & $\mathrm{F}$ & $\mathrm{F}$ & $\mathrm{F}$ & $\mathrm{F}$ & $\mathrm{F}$ & $\mathrm{F}$ \\
\hline Pericalcarine & $\mathrm{O}$ & $\mathrm{O}$ & $\mathrm{O}$ & $\mathrm{O}$ & $\mathrm{O}$ & $\mathrm{O}$ & $\mathrm{O}$ & $\mathrm{O}$ \\
\hline Postcentral & $\mathrm{P}$ & $\mathrm{P}$ & $\mathrm{P}$ & $\mathrm{P}$ & $\mathrm{P}$ & $\mathrm{P}$ & $\mathrm{P}$ & $\mathrm{P}$ \\
\hline Posterior cingulate & $\mathrm{P}$ & $\mathrm{P}$ & $\mathrm{F}$ & $\mathrm{F}$ & $\mathrm{P}$ & $\mathrm{P}$ & $\mathrm{P}$ & $\mathrm{F}$ \\
\hline Precentral & $\mathrm{F}$ & $\mathrm{F}$ & $\mathrm{P}$ & $\mathrm{P}$ & $\mathrm{P}$ & $\mathrm{P}$ & $\mathrm{P}$ & $\mathrm{P}$ \\
\hline Precuneus & $\mathrm{P}$ & $\mathrm{P}$ & $\mathrm{P}$ & $\mathrm{P}$ & $\mathrm{P}$ & $\mathrm{P}$ & $\mathrm{P}$ & $\mathrm{P}$ \\
\hline $\begin{array}{l}\text { Rostral anterior } \\
\text { cingulate }\end{array}$ & $\mathrm{F}$ & $\mathrm{F}$ & $\mathrm{F}$ & $\mathrm{F}$ & $\mathrm{F}$ & $\mathrm{F}$ & $\mathrm{F}$ & $\mathrm{F}$ \\
\hline Rostral middle frontal & $\mathrm{F}$ & $\mathrm{F}$ & $\mathrm{F}$ & $\mathrm{F}$ & $\mathrm{F}$ & $\mathrm{F}$ & $\mathrm{F}$ & $\mathrm{F}$ \\
\hline Superior frontal & $\mathrm{F}$ & $\mathrm{F}$ & $\mathrm{F}$ & $\mathrm{F}$ & $\mathrm{F}$ & $\mathrm{F}$ & $\mathrm{F}$ & $\mathrm{F}$ \\
\hline Superior parietal & $\mathrm{P}$ & $\mathrm{P}$ & $\mathrm{P}$ & $\mathrm{P}$ & $\mathrm{P}$ & $\mathrm{P}$ & $\mathrm{P}$ & $\mathrm{P}$ \\
\hline Superior temporal & $\mathrm{T}$ & $\mathrm{T}$ & $\mathrm{T}$ & $\mathrm{T}$ & $\mathrm{T}$ & $\mathrm{T}$ & $\mathrm{T}$ & $\mathrm{T}$ \\
\hline Supramarginal & $\mathrm{P}$ & $\mathrm{P}$ & $\mathrm{P}$ & $\mathrm{P}$ & $\mathrm{P}$ & $\mathrm{P}$ & $\mathrm{P}$ & $\mathrm{P}$ \\
\hline Temporal pole & $\mathrm{T}$ & $\mathrm{T}$ & $\mathrm{T}$ & $\mathrm{T}$ & $\mathrm{T}$ & $\mathrm{T}$ & $\mathrm{T}$ & $\mathrm{T}$ \\
\hline Transverse temporal & $\mathrm{T}$ & $\mathrm{T}$ & $\mathrm{T}$ & $\mathrm{T}$ & $\mathrm{T}$ & $\mathrm{T}$ & $\mathrm{T}$ & $\mathrm{T}$ \\
\hline Hemisphere & $\mathrm{L}$ & $\mathrm{R}$ & $\mathrm{L}$ & $\mathrm{R}$ & $\mathrm{L}$ & $\mathrm{R}$ & $\mathrm{L}$ & $\mathrm{R}$ \\
\hline
\end{tabular}

Notes: Abbreviations: $\mathrm{F}$ - frontal, $\mathrm{T}$ - temporal, $\mathrm{P}$ parietal, O - occipital, L - left, $\mathrm{R}$ - right. 
Table B.3. Hubs of the CT network frontal, temporal, parietal and occipital modular organization in HE, bvFTD and AD. Hubs were ranked according to their between-lobes participation index $(p)$ and within-lobe $z$-score $(z)$. First are named the left hemispheres nodes. High $p /$ high $z$ scores indicate so-called integrative regions (i.e. nodes interacting across all lobes) and regions with low $p /$ high $z$ are so-called provincial hubs (i.e. nodes which interact inside its own module/lobe).

(a) Hubs of positive sub-network

List of high $p /$ high $z$ nodes

\begin{tabular}{llllll}
\hline HE & Lobe & bvFTD & Lobe & AD & Lobe \\
\hline Caudal middle frontal & $\mathrm{F}$ & Caudal middle frontal & $\mathrm{F}$ & Rostral middle frontal & $\mathrm{F}$ \\
Superior frontal & $\mathrm{F}$ & Pars opercularis & $\mathrm{F}$ & Inferior temporal & $\mathrm{T}$ \\
$\begin{array}{l}\text { Superior parietal } \\
\text { Pericalcarine }\end{array}$ & $\mathrm{P}$ & Rostral middle frontal & $\mathrm{F}$ & Superior parietal & $\mathrm{P}$ \\
& $\mathrm{O}$ & Superior frontal & $\mathrm{F}$ & Cuneus & $\mathrm{O}$ \\
Caudal middle frontal & $\mathrm{F}$ & Cuneus & $\mathrm{O}$ & & \\
Superior frontal & $\mathrm{F}$ & Pars opercularis & $\mathrm{F}$ & Caudal middle frontal & $\mathrm{F}$ \\
Middle temporal & $\mathrm{T}$ & Pars triangularis & $\mathrm{F}$ & Superior temporal & $\mathrm{T}$ \\
Superior parietal & $\mathrm{P}$ & Rostral middle frontal & $\mathrm{F}$ & Superior parietal & $\mathrm{P}$ \\
Cuneus & $\mathrm{O}$ & Superior frontal & $\mathrm{F}$ & Cuneus & $\mathrm{O}$ \\
& & & & Lingual & $\mathrm{O}$ \\
\hline
\end{tabular}

\begin{tabular}{llllll}
\hline \multicolumn{5}{c}{ List of low $p /$ high $z$ nodes } \\
\hline HE & Lobe & bvFTD & Lobe & $\mathrm{AD}$ & Lobe \\
\hline Middle temporal & $\mathrm{T}$ & Fusiform & $\mathrm{T}$ & Lateral orbito frontal & $\mathrm{F}$ \\
Superior temporal & $\mathrm{T}$ & & & Rostral middle frontal & $\mathrm{F}$ \\
Rostral middle frontal & $\mathrm{F}$ & & & & \\
Inferior Temporal & $\mathrm{T}$ & & & & \\
\hline
\end{tabular}

(b) Hubs of negative sub-network

List of high $p /$ high $z$ nodes

\begin{tabular}{llllll}
\hline HE & Lobe & bvFTD & Lobe & AD & Lobe \\
\hline Rostral anterior cingulate & $\mathrm{F}$ & Insula & $\mathrm{F}$ & Caudal Middle Frontal & $\mathrm{F}$ \\
Middle temporal & $\mathrm{T}$ & Precentral & $\mathrm{F}$ & Insula & $\mathrm{F}$ \\
Transverse temporal & $\mathrm{T}$ & Banksts & $\mathrm{T}$ & Transverse temporal & \\
& & Transverse temporal & $\mathrm{T}$ & & \\
Rostral middle frontal & $\mathrm{F}$ & Insula & $\mathrm{F}$ & Caudal middle frontal & $\mathrm{F}$ \\
Parahippocampal & $\mathrm{T}$ & Entorhinal & $\mathrm{T}$ & Lateral orbito frontal & $\mathrm{F}$ \\
Temporal pole & $\mathrm{T}$ & Temporal pole & $\mathrm{T}$ & Temporal pole & $\mathrm{T}$ \\
\hline
\end{tabular}

Notes: Abbreviations: HE — healthy elderly, bvFTD — behavioral variant frontotemporal dementia, AD — Alzheimers disease. 
Table B.4. Hubs of the SA network frontal, temporal, parietal and occipital modular organization in HE, bvFTD and AD. Hubs were ranked according to their between-lobes participation index $(p)$ and within-lobe $z$-score $(z)$. First are named the left hemispheres nodes. High $p /$ high $z$ scores indicate so called integrative nodes (i.e. nodes interacting across all lobes) and regions with low $p /$ high $z$ are so-called provincial hubs (i.e. nodes which interact inside its own module/lobe).

(a) Hubs of positive sub-network

\begin{tabular}{|c|c|c|c|c|c|}
\hline \multicolumn{6}{|c|}{ List of high $p /$ high $z$ nodes } \\
\hline $\mathrm{HE}$ & Lobe & bvFTD & Lobe & $\mathrm{AD}$ & Lobe \\
\hline \multirow[t]{14}{*}{ Insula } & $\mathrm{F}$ & Pars orbitalis & $\mathrm{F}$ & Insula & $\mathrm{F}$ \\
\hline & & Inferior temporal & $\mathrm{T}$ & Lateral orbito frontal & $\mathrm{F}$ \\
\hline & & Middle temporal & $\mathrm{T}$ & Medial orbito frontal & $\mathrm{F}$ \\
\hline & & & & Pars orbitalis & $\mathrm{F}$ \\
\hline & & & & Rostral anterior cingulate & $\mathrm{F}$ \\
\hline & & & & Middle temporal & $\mathrm{T}$ \\
\hline & & & & Fusiform & $\mathrm{T}$ \\
\hline & & & & Postcentral & $\mathrm{P}$ \\
\hline & & & & Precuneus & $\mathrm{P}$ \\
\hline & & & & Superior parietal & $\mathrm{P}$ \\
\hline & & Middle temporal & $\mathrm{T}$ & Lateral orbito frontal & $\mathrm{F}$ \\
\hline & & Temporal pole & $\mathrm{T}$ & Banksts & $\mathrm{T}$ \\
\hline & & Precuneus & $\mathrm{P}$ & Middle temporal & $\mathrm{T}$ \\
\hline & & Pelicarcaline & $\mathrm{O}$ & Pericalcarine & $\mathrm{O}$ \\
\hline \multicolumn{6}{|c|}{ List of low $p /$ high $z$ nodes } \\
\hline $\mathrm{HE}$ & Lobe & bvFTD & Lobe & $\mathrm{AD}$ & Lobe \\
\hline Lateral orbito frontal & $\mathrm{F}$ & Pars orbitalis & $\mathrm{F}$ & & \\
\hline Pars orbitalis & $\mathrm{F}$ & Inferior temporal & $\mathrm{T}$ & & \\
\hline Inferior temporal & $\mathrm{T}$ & Middle temporal & $\mathrm{T}$ & & \\
\hline Precuneus & $\mathrm{P}$ & Temporal pole & $\mathrm{T}$ & & \\
\hline Pericalcarine & $\mathrm{O}$ & Precuneus & $\mathrm{P}$ & & \\
\hline Lateral orbito frontal & $\mathrm{F}$ & Middle temporal & $\mathrm{T}$ & & \\
\hline Precuneus & $\mathrm{P}$ & Pelicarcarine & $\mathrm{O}$ & & \\
\hline Pelicarcarine & $\mathrm{O}$ & & & & \\
\hline
\end{tabular}

(b) Hubs of negative sub-network

List of high $p /$ high $z$ nodes

\begin{tabular}{|c|c|c|c|c|c|}
\hline $\mathrm{HE}$ & Lobe & bvFTD & Lobe & $\mathrm{AD}$ & Lobe \\
\hline Pars opercularis & $\mathrm{F}$ & Caudal anterior cingulate & $\mathrm{F}$ & Frontal pole & $\mathrm{F}$ \\
\hline \multirow[t]{5}{*}{ Precuneus } & \multirow[t]{5}{*}{$\mathrm{P}$} & Rostral middle frontal & $\mathrm{F}$ & Lateral orbito frontal & $\mathrm{F}$ \\
\hline & & Banksts & $\mathrm{T}$ & Precentral & $\mathrm{F}$ \\
\hline & & Transverse temporal & $\mathrm{T}$ & Rostral anterior cingulate & $\mathrm{F}$ \\
\hline & & Inferior parietal & $\mathrm{P}$ & Pars opercularis & $\mathrm{T}$ \\
\hline & & Paracentral & $\mathrm{P}$ & Transverse temporal & $\mathrm{T}$ \\
\hline \multirow[t]{4}{*}{ Superior frontal } & \multirow[t]{4}{*}{$\mathrm{F}$} & Caudal anterior cingulate & $\mathrm{F}$ & & \\
\hline & & Lateral orbito frontal & $\mathrm{F}$ & & \\
\hline & & Rostral middle frontal & $\mathrm{F}$ & & \\
\hline & & Superior frontal & $\mathrm{F}$ & & \\
\hline
\end{tabular}

Notes: Abbreviations: HE — healthy elderly, bvFTD — behavioral variant frontotemporal dementia, AD — Alzheimer's disease. 
Table B.5. Hubs of the CT-SA coupling network frontal, temporal, parietal and occipital organisaton in HE, bvFTD and AD. Regions were ranked according to their between-lobes participation index $(p)$ and within-lobe $z$-score $(z)$. First are named the left hemispheres nodes. High $p /$ high $z$ scores indicate so-called integrative regions (that interact across all lobes).

(a) Hubs of positive sub-network

List of high $p /$ high $z$ nodes

\begin{tabular}{lllll}
\hline HE & Lobe & bvFTD & Lobe & AD \\
\hline Caudal middle frontal & $\mathrm{F}$ & Pars opercularis & $\mathrm{F}$ & Temporal pole \\
Precentral & $\mathrm{F}$ & Transverse temporal & $\mathrm{T}$ & \\
Superior frontal & $\mathrm{F}$ & Supra marginal & $\mathrm{P}$ & \\
Inferior parietal & $\mathrm{P}$ & & & \\
Inferior parietal & $\mathrm{P}$ & & & \\
Transverse temporal & $\mathrm{T}$ & & $\mathrm{F}$ & \\
Inferior parietal & $\mathrm{P}$ & Caudal middle frontal & $\mathrm{F}$ & \\
Transverse temporal & $\mathrm{T}$ & Pars opercularis & $\mathrm{F}$ & \\
& & Pars triangularis & $\mathrm{F}$ & \\
& & Superior frontal & $\mathrm{P}$ & \\
& & Post central & $\mathrm{P}$ & \\
& & Supramarginal & $\mathrm{O}$ & \\
\hline
\end{tabular}

Notes: Abbreviations: HE — healthy elderly, bvFTD — behavioral variant frontotemporal dementia, AD — Alzheimer's disease.

Table B.6. Mean cortical thickness (CT) and total surface area (SA) averaged across four lobes in each study group and global network density $\kappa$, i.e. number of links normalized to all possible number of links in the network.

\begin{tabular}{|c|c|c|c|c|c|}
\hline & Frontal & Temporal & Parietal & Occipital & Global \\
\hline & \multicolumn{4}{|c|}{$\mathrm{CT}(\mathrm{mm}) \operatorname{mean}(\mathrm{sd})$} & $\kappa$ \\
\hline $\mathrm{HE}$ & $2.56(0.09)^{\mathrm{a}, \mathrm{b}}$ & $2.67(0.02)^{\mathrm{a}, \mathrm{b}}$ & $2.22(0.02)^{\mathrm{a}, \mathrm{b}}$ & $1.85(0.03)$ & 0.13 \\
\hline bvFTD & $2.25(0.06)$ & $2.42(0.08)$ & $2.06(0.02)$ & $1.77(0.01)$ & 0.36 \\
\hline \multirow[t]{2}{*}{$\mathrm{AD}$} & $2.31(0.08)$ & $2.38(0.02)$ & $1.96(0.01)$ & $1.66(0.02)$ & 0.25 \\
\hline & \multicolumn{4}{|c|}{$\mathrm{SA}\left(\times 10^{5} \mathrm{~mm}^{2}\right)$ mean $(\mathrm{sd})$} & $\kappa$ \\
\hline $\mathrm{HE}$ & $43.4(0.5)$ & $28.9(0.6)$ & $22.2(0.9)$ & $12.3(0.8)$ & 0.10 \\
\hline bvFTD & $41.6(0.5)$ & $27.5(0.8)$ & $21.6(0.9)$ & $12.0(0.9)$ & 0.19 \\
\hline $\mathrm{AD}$ & $41.6(0.5)$ & $27.2(0.9)$ & $21.5(0.9)$ & $12.1(0.9)$ & 0.29 \\
\hline
\end{tabular}

Notes: Abbreviations: HE — healthy elderly, bvFTD — behavioral variant frontotemporal dementia, AD — Alzheimer's disease, $\kappa-$ global network density. Significant differences between raw measurements of cortical thickness (CT) and surface area (SA) between groups: a - HE/bvFTD, b - HE/AD, c - bvFTD/AD $(p<0.05)$. Statistics were calculated on regional rather than individual means. 


\section{References}

1. H.-J. Park and K. Friston, Structural and functional brain networks: From connections to cognition, Science 342 (2013) 1238411.

2. C. J. Stam, Modern network science of neurological disorders, Nat. Rev. Neurosci. 15 (2014) 683-695.

3. O. Sporns and R. F. Betzel, Modular brain networks, Annu. Rev. Psychol. 67 (2016) 613-640.

4. W. W. Seeley, R. K. Crawford, J. Zhou, B. L. Miller and M. D. Greicius, Neurodegenerative diseases target large-scale human brain networks, Neuron 62 (2009) 42-52.

5. R. L. Buckner et al., Molecular, structural, and functional characterization of alzheimer's disease: Evidence for a relationship between default activity, amyloid, and memory, J. Neurosci. 25 (2005) 77097717 .

6. J. delEtoile and H. Adeli, Graph theory and brain connectivity in alzheimers disease, Neuroscientist $\mathbf{2 3}$ (2017) 616-626.

7. J. Zhou et al., Divergent network connectivity changes in behavioral variant frontotemporal dementia and alzheimers disease, Brain 133 (2010) 13521367.

8. S. Huang et al., Learning brain connectivity of alzheimer's disease from neuroimaging data, in Advances in Neural Information Processing Systems, (2009) 808-816.

9. S. Huang et al., Learning brain connectivity of alzheimer's disease by sparse inverse covariance estimation, NeuroImage 50 (2010) 935-949.

10. S. Hulbert and H. Adeli, Eeg/meg-and imagingbased diagnosis of alzheimers disease, Rev. Neurosci. 24 (2013) 563-576.

11. F. C. Morabito et al., A longitudinal EEG study of alzheimer's disease progression based on a complex network approach, Int. J. Neural Syst. 25 (2015) 1550005.

12. W. W. Seeley, J. Zhou and E.-J. Kim, Frontotemporal dementia: What can the behavioral variant teach us about human brain organization? Neuroscientist 18 (2012) 373-385.

13. A. C. Evans, Networks of anatomical covariance, Neuroimage 80 (2013) 489-504.

14. D. S. Bassett et al., Hierarchical organization of human cortical networks in health and schizophrenia, J. Neurosci. 28 (2008) 9239-9248.

15. P. Rakic, Evolution of the neocortex: Perspective from developmental biology, Nat. Rev. Neurosci. 10 (2009) 724 .

16. A. M. Winkler et al., Cortical thickness or grey matter volume? the importance of selecting the phenotype for imaging genetics studies, Neuroimage $\mathbf{5 3}$ (2010) 1135-1146.

17. T. Jubault et al., Patterns of cortical thickness and surface area in early parkinson's disease, Neuroimage 55 (2011) 462-467.
18. A.-T. Du et al., Different regional patterns of cortical thinning in alzheimer's disease and frontotemporal dementia, Brain 130 (2007) 1159-1166.

19. G. Sanabria-Diaz et al., Surface area and cortical thickness descriptors reveal different attributes of the structural human brain networks, Neuroimage 50 (2010) 1497-1510.

20. D. S. Bassett et al., Efficient physical embedding of topologically complex information processing networks in brains and computer circuits, PLoS Comput. Biol. 6 (2010) e1000748.

21. D. S. Bassett, J. A. Brown, V. Deshpande, J. M. Carlson and S. T. Grafton, Conserved and variable architecture of human white matter connectivity, Neuroimage 54 (2011) 1262-1279.

22. F. Váša et al., Adolescent tuning of association cortex in human structural brain networks, Cereb. Cortex 28 (2017) 281-294.

23. M. C. Tartaglia, H. J. Rosen and B. L. Miller, Neuroimaging in dementia, Neurotherapeutics 8 (2011) 82-92.

24. S. Gauthier et al., Efficacy and safety of tauaggregation inhibitor therapy in patients with mild or moderate alzheimer's disease: A randomised, controlled, double-blind, parallel-arm, phase 3 trial, The Lancet 388 (2016) 2873-2884.

25. G. K. Wilcock et al., Potential of low dose leucomethylthioninium bis (hydromethanesulphonate) (lmtm) monotherapy for treatment of mild alzheimers disease: Cohort analysis as modified primary outcome in a phase iii clinical trial, $J$. Alzheimers Dis. 61(1) (2018) 435-437.

26. H. Feldman et al., A phase 3 trial of the tau and tdp43 aggregation inhibitor, leuco-methylthioniniumbis (hydromethanesulfonate)(lmtm), for behavioural variant frontotemporal dementia (bvftd), J. Neurochem. 138 (2016) 255.

27. A. D. Murray et al., The balance between cognitive reserve and brain imaging biomarkers of cerebrovascular and alzheimer's diseases, Brain 134 (2011) 3687-3696.

28. K. Rascovsky et al., Diagnostic criteria for the behavioral variant of frontotemporal dementia (bvftd): Current limitations and future directions, Alzheimer Dis. Assoc. Disord. 21 (2007) S14-S18.

29. A. M. Dale, B. Fischl and M. I. Sereno, Cortical surface-based analysis: I. segmentation and surface reconstruction, Neuroimage 9 (1999) 179-194.

30. B. Fischl and A. M. Dale, Measuring the thickness of the human cerebral cortex from magnetic resonance images, Proc. Natl. Acad. Sci. 97 (2000) 1105011055 .

31. B. Fischl et al., Automatically parcellating the human cerebral Cortex, Cereb. Cortex 14 (2004) 1122.

32. R. S. Desikan et al., An automated labeling system for subdividing the human cerebral cortex on MRI 
scans into gyral based regions of interest, Neuroimage 31 (2006) 968-980.

33. X. Han et al., Reliability of MRI-derived measurements of human cerebral cortical thickness: The effects of field strength, scanner upgrade and manufacturer, Neuroimage 32 (2006) 180-194.

34. O. Potvin, A. Mouiha, L. Dieumegarde and S. Duchesne, Effects of scanner manufacturer and strength on cortical surfaces, thicknesses and volumes in the aging brain, Alzheimers Dement. J. Alzheimers Assoc. 12 (2016) P94-P96.

35. J. Munilla et al., Construction and analysis of weighted brain networks from sice for the study of alzheimer's disease, Front. Neuroinform. 11 (2017) 19.

36. J. D. Storey, A direct approach to false discovery rates, J. R. Stat. Soc. Ser. B (Stat. Methodol) 64 (2002) 479-498.

37. B. C. van Wijk, C. J. Stam and A. Daffertshofer, Comparing brain networks of different size and connectivity density using graph theory, PLoS One $\mathbf{5}$ (2010) e13701.

38. M. Rubinov and D. S. Bassett, Emerging evidence of connectomic abnormalities in schizophrenia, J. Neurosci. 31 (2011) 6263-6265.

39. D. Meunier, S. Achard, A. Morcom and E. Bullmore, Age-related changes in modular organization of human brain functional networks, Neuroimage 44 (2009) 715-723.

40. M. P. van den Heuvel and O. Sporns, An anatomical substrate for integration among functional networks in human cortex, J. Neurosci. 33 (2013) 14489 14500 .

41. M. Xia, J. Wang and Y. He, Brainnet viewer: A network visualization tool for human brain connectomics, PloS One 8 (2013) e68910.

42. R. Romero-Garcia, M. Atienza, L. H. Clemmensen and J. L. Cantero, Effects of network resolution on topological properties of human neocortex, Neuroimage 59 (2012) 3522-3532.

43. A. Clauset, M. E. Newman and C. Moore, Finding community structure in very large networks, Phys. Rev. E 70 (2004) 066111.

44. D. J. Watts and S. H. Strogatz, Collective dynamics of small-world networks, Nature 393 (1998) 440.

45. Z. J. Chen, Y. He, P. Rosa-Neto, J. Germann and A. C. Evans, Revealing modular architecture of human brain structural networks by using cortical thickness from MRI, Cereb. Cortex 18 (2008) 23742381.

46. C. Möller et al., Joint assessment of white matter integrity, cortical and subcortical atrophy to distinguish ad from behavioral variant ftd: A two-center study, NeuroImage Clin. 9 (2015) 418-429.

47. E. Ravasz and A.-L. Barabási, Hierarchical organization in complex networks, Phys. Rev. E 67 (2003) 026112 .
48. M. Irish, O. Piguet and J. R. Hodges, Self-projection and the default network in frontotemporal dementia, Nat. Rev. Neurol. 8 (2012) 152-161.

49. M. Daianu et al., Disrupted rich club network in behavioral variant frontotemporal dementia and early-onset alzheimer's disease, Hum. Brain Mapp. 37 (2016) 868-883.

50. E. D. Huey, K. T. Putnam and J. Grafman, A systematic review of neurotransmitter deficits and treatments in frontotemporal dementia, Neurology 66 (2006) $17-22$.

51. J. T. OBrien and A. Burns, Clinical practice with anti-dementia drugs: A revised (second) consensus statement from the British association for psychopharmacology, J. Psychopharmacol. 25 (2011) 997-1019.

52. A. L. Boxer et al., Memantine in patients with frontotemporal lobar degeneration: A multicentre, randomised, double-blind, placebo-controlled trial, Lancet Neurol. 12 (2013) 149-156.

53. I. Ferrer et al., Familial behavioral variant frontotemporal dementia associated with astrocytepredominant tauopathy, J. Neuropathol. Exp. Neurol. 74 (2015) 370-379.

54. D. A. Lewis, M. J. Campbell, R. D. Terry and J. H. Morrison, Laminar and regional distributions of neurofibrillary tangles and neuritic plaques in alzheimers disease: A quantitative study of visual and auditory cortices, J. Neurosci. 7 (1987) 1799-1808.

55. P. R. Hof, C. Bouras, D. Perl and J. Morrison, Quantitative neuropathologic analysis of pick's disease cases: Cortical distribution of pick bodies and coexistence with alzheimer's disease, Acta Neuropathol. 87 (1994) 115-124.

56. C. Halabi et al., Patterns of striatal degeneration in frontotemporal dementia, Alzheimer Dis. Assoc. Disord. 27 (2013) 74.

57. A. Procter, M. Qurne and P. Francis, Neurochemical features of frontotemporal dementia, Dement. Geriatr. Cogn. Disord. 10 (1999) 80-84.

58. A. De Calignon et al., Propagation of tau pathology in a model of early alzheimer's disease, Neuron $\mathbf{7 3}$ (2012) 685-697.

59. T. Nonaka and M. Hasegawa, Tdp-43 prions, in Cold Spring Harbor Perspectives in Medicine 8(3) (2018) a024463.

60. G. Gong, Y. He, Z. J. Chen and A. C. Evans, Convergence and divergence of thickness correlations with diffusion connections across the human cerebral cortex, Neuroimage 59 (2012) 1239-1248.

61. C. L. Grady et al., Evidence from functional neuroimaging of a compensatory prefrontal network in alzheimer's disease, J. Neurosci. 23 (2003) 986-993.

62. E. A. Maguire, K. Woollett and H. J. Spiers, London taxi drivers and bus drivers: A structural MRI and neuropsychological analysis, Hippocampus 16 (2006) 1091-1101. 
63. K. Witiuk et al., Cognitive deterioration and functional compensation in ALS measured with fMRI using an inhibitory task, J. Neurosci. 34 (2014) 14260-14271.

64. W. W. Seeley et al., Divergent social functioning in behavioral variant frontotemporal dementia and alzheimer disease: Reciprocal networks and neuronal evolution, Alzheimer Dis. Assoc. Disord. 21 (2007) S50-S57.

65. L. Sommerlade et al., Inference of granger causal time-dependent influences in noisy multivariate time series, J. Neurosci. Methods 203 (2012) 173-185. 